\title{
3D imaging of cement-based materials at sub-micron resolution by combining laser scanning confocal microscopy with serial sectioning
}

\author{
M.H.N. Yio ", M.J. Mac, H.S. Wong and N.R. Buenfeld \\ Concrete Durability Group, Department of Civil and Environmental Engineering, Imperial College London, SW7 2AZ,
}

$U K$

\begin{abstract}
In this paper, we present a new method to reconstruct large volumes of non-transparent porous materials at sub-micron resolution. The proposed method combines fluorescence LSCM with serial sectioning to produce a series of overlapping confocal z-stacks which are then aligned and stitched based on phase correlation. The method can be extended in the XY plane to further increase the overall image volume. Resolution of the reconstructed image volume does not degrade with increase in sample size. We have used the method to image cementitious materials, hardened cement paste and concrete, and the results obtained show that the method is reliable. Possible applications of the method such as 3D characterisation of the pores and microcracks in hardened concrete, 3D particle shape characterisation of cementitious materials, and 3D characterisation of other porous materials such as rocks and bioceramics are discussed.
\end{abstract}

Keywords: Laser scanning confocal microscopy; 3D imaging; serial sectioning; microstructure; pore structure; microcrack; cement-based materials

\section{Introduction}

Degradation of concrete is often due to the ingress of deleterious substances such as chloride and sulphate ions in the presence of water. The ease with which fluids penetrate concrete depends predominantly on the voids present, including gel and capillary pores, microcracks and air voids, which range from a few nanometres to a few millimetres. The hydration products that form in the originally water-filled spaces are extremely heterogeneous and hence the resulting void spaces are highly tortuous and complex. It would be extremely useful to be able to characterise the pores and microcracks so as to understand better how they influence the transport properties of concrete. This would enable the development of more durable concretes and more reliable prediction of service life of concrete structures. However, direct characterisation of the pore structure and microcracks in concrete is difficult because of the complexity and range of length scale involved.

A widely used method for characterising the pore structure is mercury intrusion porosimetry. This technique measures total porosity that includes air voids and microcracks, but it underestimates pore sizes by several orders of magnitude due to the "ink-bottle" effect (Diamond, 2000). Backscattered electron imaging (BSE) in scanning electron microscopy (SEM) provides a more direct means to examine the microstructure of concrete at sub-micron resolution. The type, size and volume fraction of different phases can be measured using image analysis (Scrivener, 2004). Moreover, their chemical composition can be determined using energy-dispersive X-ray spectroscopy. However, all information obtained is limited to 2D. Three-dimensional parameters such as particle shape, connectivity and tortuosity of pores and microcracks are unattainable with this technique (Scrivener, 2004).

In the past few decades, researchers have tried various methods to characterise the three-dimensional microstructure of cement-based materials. Stutzman (1990) performed serial sectioning and imaging of cement-based materials by means of mechanical polishing combined with SEM. In his study, fiducial marks were made on the sample surface with a Knoop indenter to monitor the amount of material removed after each cycle of polishing based on the known geometry of the indenter. The specimens were small cement paste samples of about $4 \mathrm{~mm}^{2}$ impregnated with epoxy resin. Specimens were polished using $0.25 \mu \mathrm{m}$ abrasives for a few minutes, imaged, and the process repeated. The thickness of material removed each time was about 1 to $2 \mu \mathrm{m}$. Alignment of the successive layers to form a 3D image was performed in silico (Stutzman, 9 May 2014). The main challenge with the method is to ensure a small and uniform distance between each successive layer, which determines the accuracy and Z resolution of the reconstructed image. It is practically very difficult to achieve this on a large heterogeneous sample (such as concrete) and errors increase with increase in the imaged area and number of layers.

\footnotetext{
* Corresponding author: Tel: +44 (0)20 75945345.
}

E-mail address: my11@imperial.ac.uk (M.H.N. Yio). 
The development of focused ion beam nanotomography (FIB-nt) for serial sectioning with a focused Ga ${ }^{+}$ion beam combined with SEM has allowed fully automated three-dimensional imaging of cement-based materials at nano-scale resolution (Münch et al., 2006, Holzer et al., 2006, Mac et al., 2012). However, the imaged volume is small and limited by the time required for ion beam milling. To the author's knowledge, the largest FIB-nt image of a cement-based material documented in the literature is $65.7 \times 46.2 \times 33.8 \mu^{3}$ with a Z resolution of $0.17 \mu \mathrm{m}$ (Holzer et al., 2006). This size is clearly insufficient to represent mortars and concretes; hence, the technique is more feasible for localised 3D characterisation. A larger image area of $400 \times 100 \mu \mathrm{m}^{2}$ can be attained using a Xe plasma source FIB which has higher probe current and sputtering rate than $\mathrm{Ga}^{+}$FIB (Hrnčír et al., 2012). Desbois et al. (2013) have recently incorporated an argon broad ion beam polisher into a cryogenic SEM (BIB-cryo-SEM) to perform serial sectioning and imaging of sedimentary rocks. Cross sections of $2 \mathrm{~mm}^{2}$ with slice thickness (i.e. Z resolution) of $0.35 \mu \mathrm{m}$ was achieved but each slice required approximately $2 \mathrm{~h}$ of imaging time. These techniques have not been tested on cement-based materials.

X-ray microtomography ( $\mu \mathrm{CT}$ ) acquires 2D projections of the sample at many orientations and uses these to reconstruct a 3D image, thus offering non-destructive 3D imaging. $\mu \mathrm{CT}$ is able to scan sample volumes ranging from tens of $\mu \mathrm{m}^{3}$ to tens of $\mathrm{cm}^{3}$. However, its resolution decreases with increase in sample volume and is limited by the attenuation coefficient of the sample and the type of X-ray source (Hsieh, 2003). The resolution of conventional $\mu$ CT is typically > $1 \mu \mathrm{m}$ for hardened cement-based materials and therefore it has mainly been used for imaging large features such as air voids (Kim et al., 2012) and cracks (Promentilla \& Sugiyama, 2010). An improved resolution of between 0.5 to $1.0 \mu \mathrm{m}$ for cement-based materials can be achieved if synchrotron radiation is used as the X-ray source. Such systems have been used to characterise the shape of anhydrous cement particles (Garboczi \& Bullard, 2004) and pores in hardened cement paste (Gallucci et al., 2007), mortar (Sugiyama et al., 2010), concrete (Lu et al., 2006) and alkali-activated binders (Provis et al., 2012). Despite its higher resolution, Gallucci et al. (2007) reported that synchrotron $\mu$ CT is inadequate for resolving micron-sized pores at later ages (60 days) due to the loss in contrast between the pores and hydration products. Recently, synchrotron X-ray nanotomography with a spatial resolution of 30-100 nm has been reported on micron-sized samples (Provis et al., 2011, Trtik et al., 2013). In terms of practicality, access to synchrotron radiation sources is limited by the availability of facilities. Recently, new laboratory-based CTs that reportedly are able to scan samples at sub-micron and nano-scale resolutions (Lavery et al., 2014) have become available but it is unsure if such resolutions can persist on hardened cement-based materials at representative volumes.

Laser scanning confocal microscopy (LSCM) is a 3D imaging technique that has been widely used in biological research. Several authors (Lange et al., 1993, Kurtis et al., 2003, Ficker et al., 2010) have characterised surface roughness of cement-based materials using the reflectance mode of LSCM. Head and Buenfeld (2006), Head et al. (2006) and Head et al. (2008) produced 3D images of capillary pores, 'Hadley' grains and microcracks in concrete at sub-micron resolution using the fluorescence mode of LSCM. It can achieve a resolution of around $0.2 \mu \mathrm{m}$ and $0.3 \mu \mathrm{m}$ in the XY and Z planes respectively by using high numerical aperture objectives and by minimising the pinhole size (Pawley, 1995). However, the imaging depth of LSCM is extremely small for non-transparent materials because light scattering limits laser penetration and degrades the obtained fluorescence signal. An imaging depth of $\sim 7 \mu \mathrm{m}$ for concrete at $0.3 \mu \mathrm{m} \mathrm{Z}$ resolution (Head \& Buenfeld, 2006) and $~ 90-100 \mu \mathrm{m}$ for sandstone (Petford et al., 2001) and marble (Mauko et al., 2009) at $2 \mu \mathrm{m} \mathrm{Z}$ resolution has been reported.

The definition of resolution in the literature can be somewhat confusing. Spatial resolution and voxel resolution are the two main terms that are often used interchangeably to mean the resolving power of an imaging system, but in fact they carry two very different meanings. Spatial resolution is the minimum separation between two points that can be resolved whereas voxel resolution refers to the total number of voxels or the voxel size that is sampled by an imaging system. Therefore, spatial resolution is comparably more meaningful than voxel resolution. Most literature, particularly those concerned with X-ray CT and FIB-nt, often report only the voxel resolution.

Table 1 summarises the main 3D imaging techniques that have been used to characterise cement-based materials over the last decade. The list of references is not exhaustive, but gives an idea of the capabilities, advantages and limitations of these techniques for characterising cement-based materials. Note that the resolutions given for FIB-nt and X-ray CT are voxel resolution (some have been ambiguously reported as spatial resolution, but in terms of $\mu \mathrm{m} / \mathrm{voxel}$ ) and those for LSCM are spatial resolution. Although LSCM has better resolution than X-ray $\mu C T$ and images a larger area than FIB-nt, it has a small imaging depth and this limits its ability to image a representative volume of cement-based materials. In biological imaging, thick tissues can be cut into serial sections of equal thickness by means of microtome (Čapek et al., 2009) or cryostat sectioning (Luzzati et al., 2011) and then imaged sequentially by LSCM to form a large 3D image volume. However, this technique causes material loss between slices and is not applicable to cement-based materials.

In this paper, we propose a new method that enhances the ability of LSCM to image large 3D volumes of porous materials, such as cement-based materials, at sub-micron resolutions. In the proposed method, LSCM is combined with serial sectioning to image successive z-stacks. The key feature of the method is that each stack has a small overlapping volume with neighbouring stacks, thus allowing them to be stitched together using a phase correlation function to progressively form a large 3D image without loss of information between stacks. This can be extended in the XY direction to image large samples. To some extent, the proposed method bridges a critical gap between $\mathrm{X}$-ray $\mu \mathrm{CT}$ and 
FIB-nt in terms of the resolution and imaged volume to characterise capillary pores and microcracks in concrete. We have trialled the method on fly ash, cement paste and concrete samples. The results obtained show that the proposed method is viable. The advantages and limitations of the proposed method, as well as its potential applications are discussed. 


\begin{tabular}{|c|c|c|c|c|c|c|c|}
\hline \multicolumn{3}{|c|}{ Imaging technique } & References & $\begin{array}{l}\text { Image/Sample } \\
\text { volume }\left(\mu \mathrm{m}^{3}\right)\end{array}$ & Resolution $(\mu \mathrm{m}) *$ & Advantages & Disadvantages \\
\hline \multirow{3}{*}{\multicolumn{3}{|c|}{$\begin{array}{l}\text { Focused Ion Beam - } \\
\text { Nanotomography } \\
\text { (FIB-nt) }\end{array}$}} & Holzer et al. (2006) & $5.2 \times 5.4 \times 2.9$ & $0.0085 \times 0.0108 \times 0.017$ & \multirow{3}{*}{$\begin{array}{l}\text { - Very high resolution }(\sim 10 \mathrm{~nm}) \\
\text { - Compositional imaging in BSE mode } \\
\text { allows phases with different atomic number } \\
\text { to be differentiated. }\end{array}$} & \multirow{3}{*}{$\begin{array}{l}\text { - Extremely small image volume } \\
\text { - Long acquisition time for serial sectioning } \\
\text { - Sample preparation may cause microstructural } \\
\text { damage and unevenly milled surfaces. } \\
\text { - Relatively expensive } \\
\text { - Not practical for concrete }\end{array}$} \\
\hline & & & Holzer et al. (2007) & & $0.0198 \times 0.0251 \times 0.04$ & & \\
\hline & & & Holzer et al. (2006) & $65.7 \times 46.2 \times 33.8$ & $0.0849 \times 0.1077 \times 0.17$ & & \\
\hline \multirow{12}{*}{ 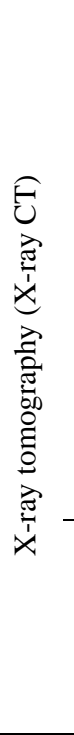 } & \multirow{9}{*}{ 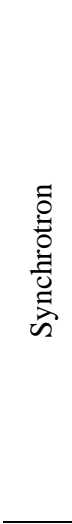 } & \multirow[b]{2}{*}{ Nano } & Provis et al. (2011) & Micron-sized & 0.03 & \multirow{12}{*}{$\begin{array}{l}\text { - Large image volume is possible } \\
\text { - Non-destructive imaging } \\
\text { - Minimal sample preparation } \\
\text { - Phases can be differentiated based on } \\
\text { their composition and density } \\
\text { - Imaging in ambient environment }\end{array}$} & \multirow{12}{*}{$\begin{array}{l}\text { - Resolution decreases with increase in sample size } \\
\text { (for voxel resolution } \sim 0.5 \mu \mathrm{m} \text {, sample size is } \\
\text { normally around } 1 \mathrm{~mm} \text { ) } \\
\text { - Access to synchrotron radiation is limited by } \\
\text { availability of facilities and hence synchrotron X-ray } \\
\text { tomography is not suitable for routine imaging }\end{array}$} \\
\hline & & & $\begin{array}{l}\text { Trtik et al. (2013) } \\
\text { (Ptychographic) }\end{array}$ & $30(\varnothing) \times \mathrm{h}$ & 0.0436 & & \\
\hline & & \multirow{7}{*}{ Micro } & Sugiyama et al. (2010) & $\leq 1000^{3}$ & 0.5 & & \\
\hline & & & Gallucci et al. (2007) & $580(\varnothing) \times \mathrm{h}$ & 0.6835 & & \\
\hline & & & Provis et al. (2012) & $\leq 1000^{3}$ & 0.75 & & \\
\hline & & & Bentz et al. (2002) & $1000(\varnothing) \times \mathrm{h}$ & 0.95 & & \\
\hline & & & Lu et al. (2006) & $\leq 750(\varnothing) \times \mathrm{h}$ & 1 & & \\
\hline & & & Monteiro et al. (2009) & $\leq 250(\varnothing) \times \mathrm{h}$ & 1.54 & & \\
\hline & & & Rougelot et al. (2010) & $\begin{array}{c}8000(\varnothing) \times 20000 \\
-30000 \\
\end{array}$ & 5.3 & & \\
\hline & \multirow{3}{*}{\multicolumn{2}{|c|}{ Non-synchrotron }} & Dauzères et al. (2014) & $1000^{3}$ & 1 & & \\
\hline & & & Kim et al. (2012) & $\begin{array}{c}12000(\varnothing) \times \\
10000\end{array}$ & 10.8 (XY); $8.7(\mathrm{Z})$ & & \\
\hline & & & $\begin{array}{l}\text { Promentilla and Sugiyama } \\
\text { (2010) }\end{array}$ & $\begin{array}{c}12000(\varnothing) \times \\
24000\end{array}$ & $12(\mathrm{XY}) ; 40(\mathrm{Z})$ & & \\
\hline \multirow{2}{*}{\multicolumn{3}{|c|}{$\begin{array}{l}\text { Laser scanning confocal } \\
\text { microscopy (LSCM) }\end{array}$}} & Kurtis et al. (2003) & $\begin{array}{c}(\text { Not available })^{2} \times \\
18.2\end{array}$ & $\begin{array}{c}0.18(\mathrm{XY}) ; 0.30(\mathrm{Z}) \\
{[100 \times \text { objective }]}\end{array}$ & \multirow{2}{*}{$\begin{array}{l}\text { - Sub-micron resolution at large field of } \\
\text { views (several hundreds of microns) } \\
\text { - Shorter acquisition time } \\
\text { - Non-invasive optical sectioning } \\
\text { - Imaging in ambient environment }\end{array}$} & \multirow{2}{*}{$\begin{array}{l}\text { - Limited subsurface imaging depth } \\
\text { - Significantly poorer resolution compared to FIB-nt } \\
\text { - Sample preparation may cause microstructural } \\
\text { damage } \\
\text { - Images only fluorescent resin filled pores and } \\
\text { cracks in fluorescence mode (can be an advantage if } \\
\text { interconnected pores are of interest) }\end{array}$} \\
\hline & & & Head and Buenfeld (2006) & $140^{2} \times 7$ & $\begin{array}{l}0.18(\mathrm{XY}) ; 0.30(\mathrm{Z}) \\
\quad[63 \times \text { objective }]\end{array}$ & & \\
\hline
\end{tabular}

* Note that the resolution reported in this table for FIB-nt and X-ray CT are the voxel resolution and those for LSCM are spatial resolution.

Table 1: Review of main 3D imaging techniques used for characterising cement-based materials. 


\section{Laser scanning confocal microscopy}

The principle of LSCM for three-dimensional imaging is explained in detail in Pawley (1995) and Head and Buenfeld (2006). Fundamentally, a highly focused laser beam is used as a light source and a pinhole is employed to remove outof-focus light emanated from below and above the focal plane so as to give a very small focal volume. By scanning the light source across the sample and into the sample by raising or lowering the microscope stage (or objective lens), a series of two-dimensional images is obtained at various depths within the sample. A set of such images from successive focal planes is known as a z-stack and is used to generate a high-resolution 3D image. Thus, LSCM is a direct and noninvasive means of 3D imaging by serial optical sectioning.

Diffraction of light occurs when the laser beam passes through the sample and optics in the system; as a consequence, the image formed is a spread Airy disk with a series of concentric rings around a central spot of high intensity. The resolution of LSCM is therefore diffraction limited. The degree of spreading is termed as the point spread function (PSF). Due to the elongated shape of the PSF along the optical axis, the $\mathrm{Z}$ resolution is usually 2 or 3 times worse than the XY resolution. The XY resolution, according to the Rayleigh criterion, is the distance between two points at onehalf the Airy disk diameter (Pawley, 1995). The Airy disk diameter depends on the excitation wavelength $(\lambda)$ and the numerical aperture (NA) of the objective lens. The $\mathrm{Z}$ resolution, which is also the optical section thickness, is also dependent on the refractive index of immersion oil $\left(\mathrm{n}_{1}\right)$ and the pinhole aperture size $(\mathrm{P})$. The excitation wavelength is predetermined by the fluorophores used, while the numerical aperture and immersion oil are fixed to the objective used. The $\mathrm{Z}$ resolution can be significantly improved by reducing the pinhole size. For high power objectives such as $40 \times$, $63 \times$ and $100 \times$ oil immersion objectives with NA $>1.0$, the XY and $\mathrm{Z}$ resolution can be $\leq 0.2 \mu \mathrm{m}$ and $\leq 0.5 \mu \mathrm{m}$ respectively when the pinhole aperture is reduced to around 0.3 Airy unit . One Airy unit defines the diameter of the first concentric ring in the Airy disk (Pawley, 1995). There are many formulae in the literature for calculating the XY and $\mathrm{Z}$ resolutions of LSCM and different manufacturers use slightly different approaches to define the resolutions of their systems. The following two expressions are given by Leica Microsystems Inc. for the system used in this study:

$$
\begin{gathered}
\text { XY resolution }=0.4 \frac{\lambda}{N A} \\
\text { Z resolution }=\sqrt{\left[\left(\frac{\lambda n_{1}}{N A^{2}}\right)^{2}+\left(\frac{1.22 \sqrt{2} P n_{1} \lambda}{N A^{2}}\right)^{2}\right]}
\end{gathered}
$$

Pores and microcracks in hardened cement-based materials can only be imaged if they contain fluorophores, this is achieved by vacuum-impregnating the sample with a fluorescent dyed epoxy resin (Head \& Buenfeld, 2006, Wong \& Buenfeld, 2006). It is noted that vacuum impregnation is able to fill very fine pores with epoxy resin for BSE imaging, which has a greater resolving power than LSCM and so this does not pose a limitation. Fluorophores have their corresponding maximum absorption and emission wavelengths; therefore, it is crucial to ensure that the correct laser wavelength and beam splitter are used for imaging. It has been demonstrated in Head and Buenfeld (2006) and Head et al. (2006) that LSCM images of capillary pores in the XY plane are comparable to those obtained using BSE. However, cement-based materials contain various translucent and opaque components such as hydration products, anhydrous cement grains and aggregates. This limits the laser penetration depth and the ability of LSCM to image a representative volume.

\section{Preliminary trials}

Prior to the development of the proposed method, various methods to optimise and enhance the imaging depth of fluorescence LSCM without serial sectioning were trialled (see Section 5.1 for materials and sample preparation and Section 5.3 for details of the confocal microscope used). These include:

- Optimisation of fluorophore concentration to prevent self-quenching of fluorophores which can result in reduced fluorescence intensities. Details are presented in Section 5.1. Results show that a low fluorophore concentration (0.05 wt. \%) was sufficient to give the maximum fluorescence intensity under normal operating laser intensity of $15 \%$. Varying the fluorophore concentration did not produce a significant improvement in imaging depth.

- $\quad$ Light compensation by means of increasing the sensitivity of photomultiplier tube (PMT) or increasing laser intensity with imaging depth. These compensations are linear, i.e. not according to the exponential decay of signal with increasing depth and they were found to improve mainly the brightness of images. Compensation by laser intensity was found to improve the signal-to-noise ratio, but the imaging depth was only increased by a marginal fraction.

- Image processing methods such as histogram matching performed with Fiji (Schindelin et al., 2012) and blind deconvolution performed with AutoDeblur (Media Cybernatics). Histogram matching modifies the histograms 
of successive image slices from deeper sections to match that of the first surface image (Castleman, 1996). This equalises image intensities throughout the stack, but the clarity and sharpness of images remain unchanged and degrade with depth. Blind deconvolution improves the resolution of optical images by removing out-of-focus light based on a theoretically generated PSF (Pawley, 1995). We observed that blind deconvolution improved overall image quality, particularly those near the sample surface. The quality of images from deeper sections remains relatively poor.

- Two-photon microscopy which uses pulsed laser of longer wavelengths to reduce light scattering and to achieve deeper imaging depth. This investigation was carried out with a Leica TCS SP5 equipped with a tuneable infrared laser. However, results showed that the achievable imaging depth was similar to that of conventional LSCM as no significant improvement was observed.

\section{Proposed method}

Given that none of the methods explored in Section 3 were successful at increasing the imaging depth of LSCM, we developed a new method to obtain large 3D volumes of hardened cement-based materials by serial sectioning, sequential imaging and reconstruction. An important feature of the method is that the z-stack from each serial section shares overlapping regions with the subsequent section for stitching using 3D image reconstruction tools. Prior to serial sectioning, the samples are impregnated with fluorescent epoxy resin (see Section 5.1) to preserve the microstructure and more importantly to ensure the visibility of interconnected void space. The method consists of 5 main steps as follows:

i. Serial sectioning - This is done by mechanical grinding/polishing to expose new surfaces for imaging. Cementbased materials are highly heterogeneous and contain phases of varying hardness, so the time and grinding media required to remove a certain amount of material may vary from sample to sample. Hence, an initial process of trial and error is required to estimate these. This is crucial because the success of the method relies on the ability to control the amount of material removed and to ensure that each consecutive z-stack contains overlapping regions for stitching. Generally, polycrystalline diamonds of $15 \mu \mathrm{m}$ can be used but the grinding time should be limited to a few seconds per direction (see Section 5.2). The force exerted on the sample and the rotation frequency of the plate should be kept moderately low (e.g. $7 \mathrm{~N}$ and $50 \mathrm{RPM}$ ) to prevent over grinding. It should be stressed that these are not definitive. Slight adjustments may be required to accommodate variations in hardness of the sample along the sectioning depth.

ii. Image acquisition - Following each cycle of sectioning, a high power objective with sub-micron resolution is used to acquire a z-stack from a plane slightly above the sample surface to a plane well below it. The thickness of the stack depends on the type of material imaged and the objective used. For hardened concrete and cement pastes imaged with either a $40 \times$ or $63 \times$ objectives, z-stacks of approximately $10 \mu \mathrm{m}$ thick ensures that each contains a sufficient number of well-focused optical slices that can be used for 3D reconstruction. The intensity profile provides a good indication of where the image acquisition should begin and end because image intensity is high near the surface and gradually fades away from the surface (Fig. 1). If a larger area is required, mosaic of image tiles can be acquired.

iii. Image selection - Well-focused images with high signal/noise ratio are selected from each stack for stitching. This is done based on the intensity profiles of each stack (Fig. 1), which always show a peak corresponding to some point below the sample surface. Images within 2.0 to $3.0 \mu \mathrm{m}$ above and below the peak intensity can be selected depending on the sample and the imaging settings used. The number of images selected from each stack is kept constant.

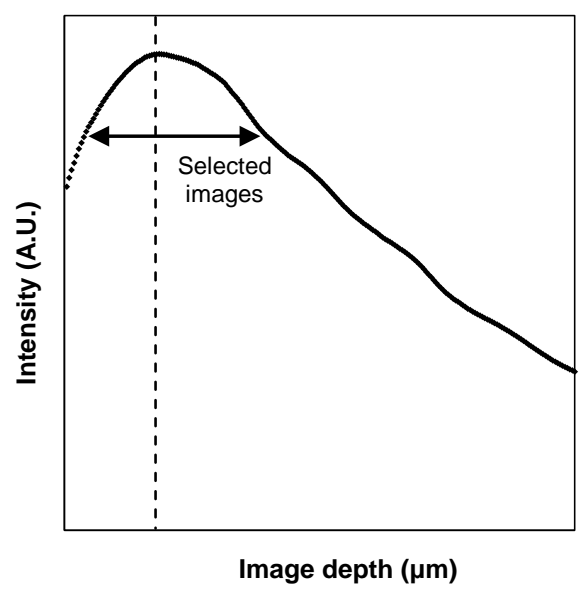

Fig. 1. Intensity profile of images from a single z-stack showing signal degradation with increase in depth below sample surface. Well-focused images are selected for subsequent stitching. 
iv. Alignment - The selected images are then aligned using image registration. Here, we use StackReg (Thevenaz et al., 1998) in Fiji which is based on an automatic sub-pixel registration algorithm that minimises the mean square difference of intensities between a reference and a test data set. It uses each slice as a template with respect to which the next slice is aligned, so that the alignment proceeds by propagation. Since the sample is well-preserved with epoxy resin, deformation does not occur during serial sectioning. Hence, the alignment process is entirely based on rigid transformation that involves only translation and rotation of images.

v. Stitching - Finally, the aligned z-stacks are stitched. Stitching is done in pairs between consecutive stacks (e.g. stack 1 with stack 2, etc.) using the Pairwise stitching plugin (Preibisch et al., 2009), which calculates the translational shifts in the $\mathrm{X}, \mathrm{Y}$ and $\mathrm{Z}$ directions between two overlapping z-stacks based on Fourier transformbased phase correlation. Note that rotational shift has been accounted for during the image alignment process (see step iv). Once the translational shifts are determined, the two z-stacks are fused together based on nonlinear blending that minimises the brightness difference across the boundary. All the z-stacks stitched in pairs are then progressively stitched together based on the computed translational shifts to reconstruct a final 3D image. If mosaic tiles are acquired, they should be stitched in $\mathrm{XY}$ prior to the stitching in the $\mathrm{Z}$ direction and this can be easily done using the Grid/Collection stitching plugin (Preibisch et al., 2009). The stitching processes are automated, and able to compute the translational shifts at sub-pixel precision. Accuracy is expressed in terms of correlation coefficient, $\mathrm{R}(0 \leq \mathrm{R} \leq 1)$ where $\mathrm{R}=1$ indicates that the overlapping regions from each z-stack are perfectly identical and hence are highly correlated.

Fig. 2 shows a schematic illustration of the stitching process. Fig. 3 shows an actual demonstration of the process on fly ash embedded in epoxy. The images are cropped to highlight a small region of interest in the XZ plane. The first column shows eight stacks, each stack is $4.45 \mu \mathrm{m}$ thick and consists of 30 images. The second column shows the same z-stacks after alignment, but the fly ash particles appear ovate due to presence of overlapping regions. The third column shows the reconstructed image where the stacks are stitched at the overlapping regions. The fly ash particles now appear to be spherical. However, uneven brightness may occur as a result of slight variations in the imaging conditions, sample characteristics and/or photo-bleaching. This can be corrected by histogram matching. Fig. 4 shows a close-up of the stitching outcome between z-stack number 3 and 4 from Fig. 3 . The overlapping region between the two stacks is marked yellow. The displacement between the two stacks in $\mathrm{Z}$, termed as axial shift $(\Delta \mathrm{Z})$, is also annotated. $\Delta \mathrm{Z}$ provides an indication of the thickness of materials removed by sectioning.

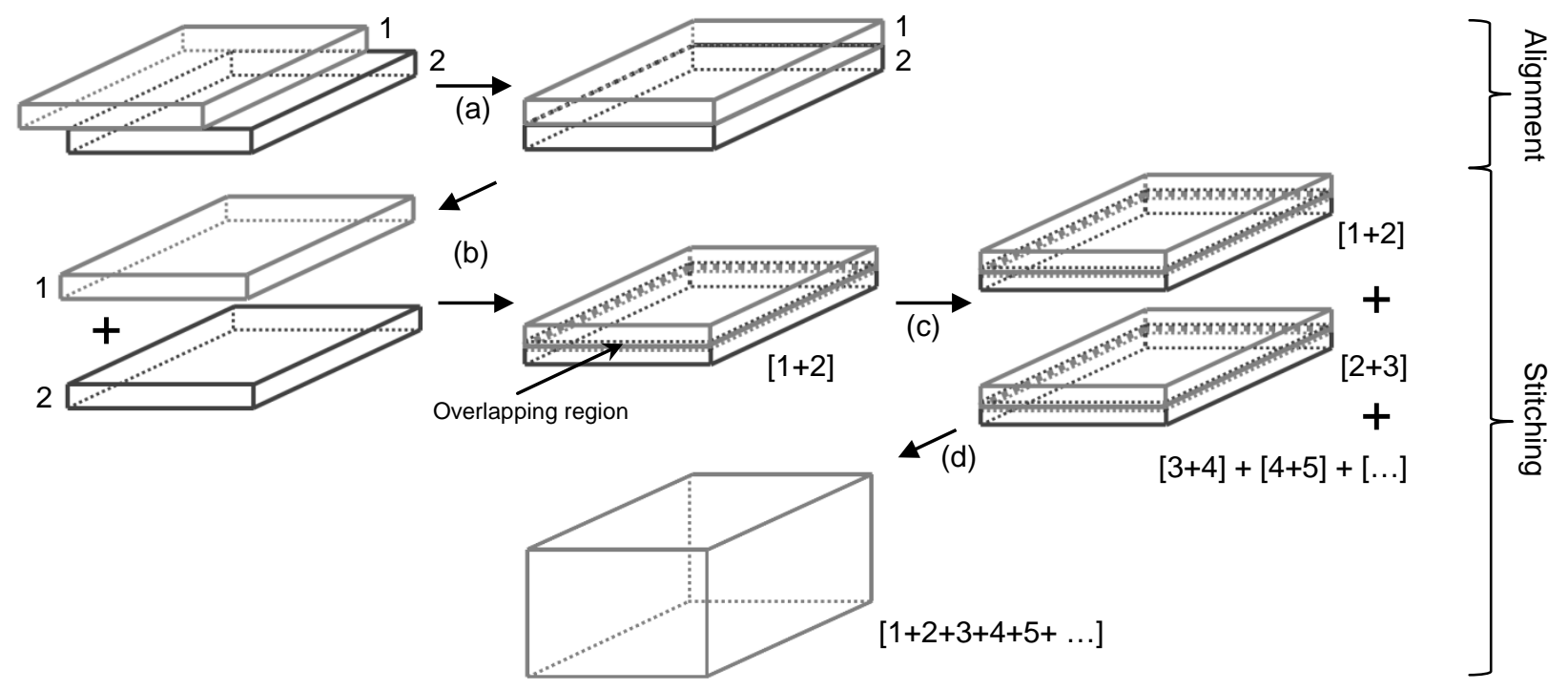

Fig. 2. Schematic diagram of the stitching process. Selected images from each z-stack are aligned (A), stitched in pairs (B), and then progressively combined (i.e. $[1+2]+[2+3]+[3+4]+[4+5]+[\ldots])$ by stitching the overlapping regions $(C)$ to reconstruct an image volume (D). 


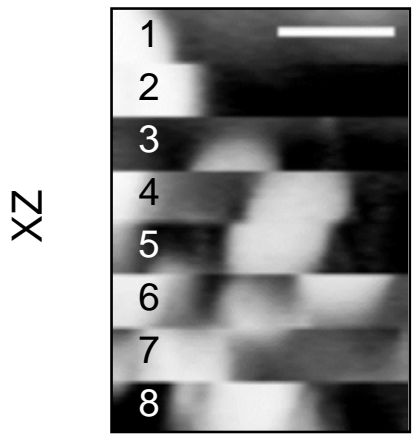

(a)

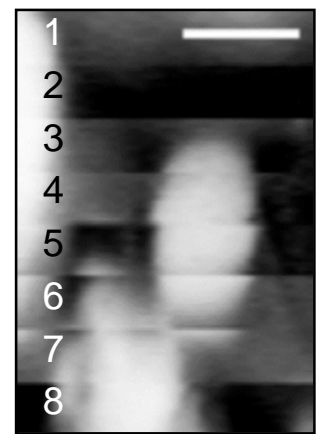

(b)

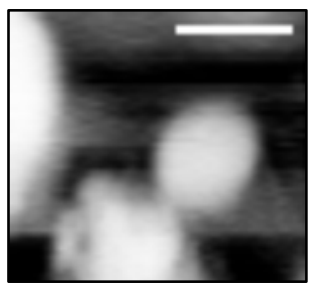

(c)

Fig. 3. Demonstration of the stitching process on fly ash embedded in resin: (A) selected images from eight serial section z-stacks, (B) alignment of the image stacks and (C) stitching of the overlapping regions. Scale bar represents $10 \mu \mathrm{m}$.

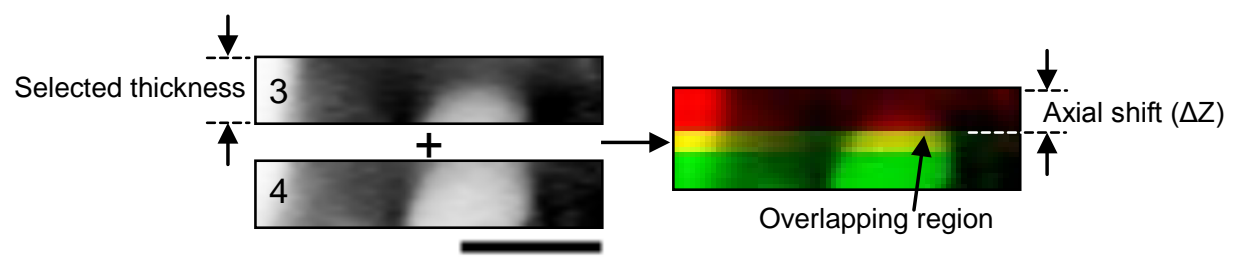

Fig. 4. Close-up of the stitching outcome between z-stack number 3 and 4 from Fig. 3 . Scale bar is $10 \mu \mathrm{m}$.

\section{Experimental}

\subsection{Materials and sample preparation}

The method described above was applied on unreacted fly ash, CEM I cement paste and concrete samples. The epoxy resin used was Struers Epofix ${ }^{\circledR}$ which has a refractive index of 1.578. The fluorophore was Struers EpoDye (C.I. Solvent Yellow 43) that has a maximum absorption wavelength and maximum emission wavelength of $490 \mathrm{~nm}$ and 520 nm respectively (see Fig. 5 (A)). These are similar to those of fluorescein. To establish the optimum concentration of fluorophore, epoxy mixtures containing 0.001 to $5 \mathrm{wt}$. \% fluorophore were prepared and mixed with a magnetic stirrer for $24 \mathrm{~h}$ to ensure that the fluorophores were evenly dispersed in the resin. Droplets of the epoxy were then excited using a $488 \mathrm{~nm}$ laser at intensities of 1\%, 4\% and 15\% (i.e. the default setting for fluorescein). The PMT was set at a constant $625 \mathrm{~V}$. It was found that using the default laser intensity of $15 \%$, a 0.05 wt. \% dye concentration was sufficient to give the maximum fluorescence intensity (see Fig. 5(B)). This value was therefore taken as the optimum concentration.
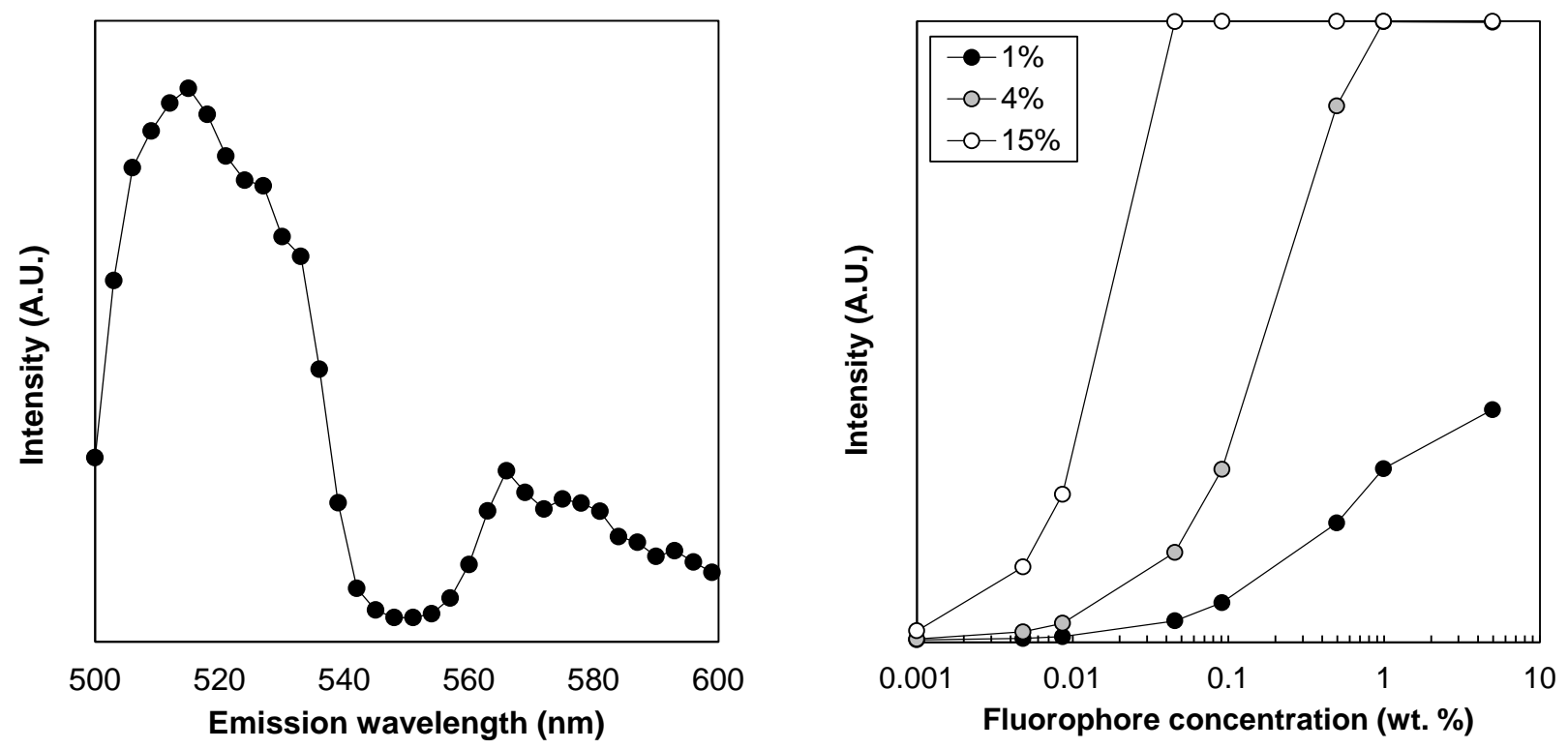
Fig. 5. (A) Emission profile of EpoDye; (B) Plot showing fluorescence intensity versus concentration of EpoDye (excited by a $488 \mathrm{~nm}$ laser at intensities of $1 \%, 4 \%$ and $15 \%$ ).

The fly ash used was type F complying with BS EN 450. Two samples were prepared in two different ways. The first sample was prepared by compacting dry fly ash particles in a small rubber mould of $2.5 \times 5 \times 1 \mathrm{~cm}^{3}$, followed by vacuum impregnation with fluorescent epoxy resin. The second sample was prepared by hand-mixing dry fly ash particles with fluorescent epoxy resin at a fly ash-epoxy mass ratio of 1.0. The mixture was cast in a separate rubber mould, de-aired in vacuum desiccator and left to set overnight. The second procedure allowed the fly ash to be suspended in the resin so that individual particles can be imaged and used to assess the accuracy of the 3D reconstruction based on their spherical shape. It was however noted that such preparation could cause the grinding surface to have variable hardness which would in turn increase the likelihood of surface relief and scratches.

The cement paste was prepared using Portland cement CEM I complying with BS EN 197-1 and tap water mixed at a water/cement (w/c) ratio of 0.30 in a bowl mixer. The fresh cement paste was compacted in two equal layers into a plastic cylindrical mould (58 mm diameter, $49 \mathrm{~mm}$ height) and rotated. After the initial $24 \mathrm{~h}$, the cement paste was wrapped in cling film and sealed in a polythene bag at $20^{\circ} \mathrm{C}$ until the age of 3 days. A rectangular block sample ( $40 \times$ $20 \times 8 \mathrm{~mm}$ ) was then sectioned from the mid-height of the cylinder, freeze-dried to remove pore water and impregnated with fluorescent epoxy resin following the method described in Wong and Buenfeld (2006).

The concrete sample was prepared with Portland cement CEM I, Thames Valley sand ( $<5 \mathrm{~mm})$, gravel $(5-10 \mathrm{~mm})$ and tap water at a w/c ratio of 0.5 . The total aggregate fraction was $60 \%$ and the sand to total aggregate ratio was 0.4 . The fresh mix was cast into a cylindrical mould (100 mm diameter, $50 \mathrm{~mm}$ height) and compacted on a vibrating table. After the initial $24 \mathrm{~h}$, the sample was demoulded and cured under water at $20^{\circ} \mathrm{C}$. At the age of 14 days, the sample was exposed to 56 cycles of freeze-thaw action in accordance with CEN/TR 15177:2006. The purpose of this was to produce microcracks within the sample. Following that, the sample was gently dried at stepwise temperatures of $30^{\circ} \mathrm{C}$, $40^{\circ} \mathrm{C}$ and $50^{\circ} \mathrm{C}$ until moisture equilibrium was reached to prevent additional cracking. The sample was then impregnated with fluorescent epoxy resin under 7 bar pressure and sectioned to extract a rectangular block $(40 \times 20 \times 8$ $\mathrm{mm}$ ) for imaging. The chemical and physical properties of the fly ash and Portland cement are presented in Table 2.

Table 2: Physical and chemical properties of Portland cement and fly ash used.

\begin{tabular}{lcc}
\hline & Portland cement & Fly ash \\
\hline Chemical analysis (wt. \%) & & \\
$\mathrm{CaO}$ & 65.0 & 0.1 \\
$\mathrm{SiO}_{2}$ & 20.5 & 72.2 \\
$\mathrm{Al}_{2} \mathrm{O}_{3}$ & 5.0 & 24.3 \\
$\mathrm{Fe}_{2} \mathrm{O}_{3}$ & 2.7 & 0.4 \\
$\mathrm{MgO}$ & 0.9 & 0.1 \\
$\mathrm{Na} 2$ & 0.19 & 0.3 \\
$\mathrm{~K}_{2} \mathrm{O}$ & 0.73 & - \\
$\mathrm{SO}_{3}$ & 3.1 & 0.1 \\
$\mathrm{Cl}^{-}$ & 0.01 & - \\
& & \\
Physical analysis & & \\
Specific gravity & 3.15 & 2.25 \\
$\mathrm{Blain}$ surface area $\left(\mathrm{m}^{2} / \mathrm{kg}\right)$ & 342 & - \\
Laser granulometry $\mathrm{d}_{50}(\mu \mathrm{m})$ & - & 7 \\
Loss on ignition $(\%)$ & 1.36 & - \\
\hline
\end{tabular}

\subsection{Serial sectioning}

Serial sectioning was performed by grinding using a resin bonded diamond disc at 1200 grit / $15 \mu$ fineness (Struers MD-Piano on a LaboPol-5 machine). The force exerted on the samples was maintained at $7 \mathrm{~N}$ and the rotation frequency of the plate was maintained at 50 RPM. Grinding was carried out for a very short period to control the amount of material removed between each section. The average optimum grinding times were determined to be around $1 \mathrm{~s}$ per direction for the fly ash-epoxy blocks, $2 \mathrm{~s}$ per direction for the cement paste and 2 to $3 \mathrm{~s}$ per direction for the concrete. This removed approximately 1.8 to $3.3 \mu \mathrm{m}$ thickness of material from the samples (see Section 6.2). Following this, the samples were polished using diamond at successively finer sizes of 9, 6, 3 and $1 \mu$ m fineness (3 to 4 minutes per direction) for imaging.

\subsection{LSCM}

A Leica TCS SP5 laser scanning confocal microscope was used for imaging. The microscope is equipped with a Märzhäuser motorised scanning stage with a resolution (stepping distance) of $0.025 \mu \mathrm{m}$ and a repeatability of $\pm 0.75 \mu \mathrm{m}$. 
An argon laser line of $488 \mathrm{~nm}$ was used to excite the fluorophores and the beam splitter was configured to range from $500 \mathrm{~nm}$ to $600 \mathrm{~nm}$ to allow the emitted fluorescence to be collected by the PMT. A 40× (NA 1.25) oil immersion objective with a spatial XY resolution of $0.156 \mu \mathrm{m}$ and a field of view of $387 \times 387 \mu \mathrm{m}^{2}$ was used in all cases.

Based on the Nyquist theorem, the smallest feature that can be resolved is either $2.3 \times$ the pixel size or the spatial resolution, whichever is larger (Pawley, 1995). This also applies to the voxel spacing in Z. Mismatch of refractive indices between the objective immersion oil and epoxy resin can cause axial geometrical distortion to LSCM images because of the shift in focus position when the laser travels from the immersion oil into the epoxy resin. It is crucial that such distortion is corrected if quantitative data is to be extracted from the images. In our study, the refractive index of the immersion oil $\left(\mathrm{n}_{1}=1.518\right)$ is smaller than that of the epoxy resin $\left(\mathrm{n}_{2}=1.578\right)$, resulting in axial compression to the image volume. This can be corrected to $0.5 \%$ accuracy using the linear correction method proposed by Van Elburg et al. (2007):

$$
A S F-1=1.132 \frac{\left(n_{2}-n_{1}\right)}{n_{1}}+0.0065 \tan ^{-1}\left(100 \frac{\left(n_{2}-n_{1}\right)}{n_{1}}\right)
$$

Where ASF is the axial scaling (correction) factor. Given that $n_{1}=1.518$ and $n_{2}=1.578$, ASF $=1.051$. Multiplying the voxel spacing by the ASF gives the corrected image thickness.

The imaging settings for each sample are summarised in Table 3. The hand-mixed fly ash sample (for validation purpose) and the cement paste sample were both imaged at a higher resolution by reducing the pinhole size to 0.3 Airy unit and increasing the zoom factor to $1.8 \times$. Images were digitised to $2048 \times 2048$ pixels to give a pixel spacing of 0.105 $\mu \mathrm{m}$. The vacuum-impregnated fly ash sample was intended for particle shape characterisation. Therefore, the same pinhole size was used, but a lower zoom factor of $1.46 \times$ was adopted to capture a larger number of particles. The images were digitised to $1024 \times 1024$ to give a pixel spacing of $0.26 \mu \mathrm{m}$ to match with the voxel spacing required to resolve details in $\mathrm{Z}$ at the optimised resolution of $0.534 \mu \mathrm{m}$. The voxel size could be made smaller in both $\mathrm{XY}$ and $\mathrm{Z}$ but this would increase the scanning time. Moreover, this would not provide additional information for the purpose of particle shape characterisation since the minimum particle size that can be characterised is limited by the $\mathrm{Z}$ resolution. For the concrete sample, the default imaging settings ( 1 Airy unit pinhole, $1 \times$ zoom) were used and images were digitised to $1024 \times 1024$ pixels at a pixel spacing of $0.378 \mu \mathrm{m}$. This allows us to image a larger volume of the sample at a sufficient resolution to image the microcracks. The laser intensity was $20 \%$ for the fly ash and cement paste, and $15 \%$ for the concrete. The PMT was adjusted correspondingly to prevent under-saturation or over-saturation of the brightness intensity.

When the pinhole size is reduced to less than 1 Airy unit, the noise level of images could significantly increase. The noise level can be reduced by performing line or frame averaging during the scanning process to scan each pixels several times and averaging the scans. However, the scanning time would be significantly prolonged. Alternatively, post image processing methods such as median or Gaussian filtering can be applied to reduce the noise of the acquired images. The second approach was mainly adopted in this study (for the vacuum-impregnated fly ash sample, two-times line averaging was also applied).

Serial sectioning and imaging were repeated on the cement paste and concrete samples until the reconstructed thickness was $>100 \mu \mathrm{m}$ whilst for the hand-mixed and vacuum-impregnated fly ash samples; the process was repeated until the reconstructed thickness was similar to that of a $15 \mu \mathrm{m}$ thick reference image and about $50 \mu \mathrm{m}$, respectively. The reference image for the hand-mixed sample was acquired prior to the start of the sectioning process and this will be used for comparison with the reconstructed 3D image. The concrete sample was also imaged in a $2 \times 2$ mosaic to increase the image volume further in the XY plane (see Section 7. Discussion).

Table 3: Imaging settings for each sample.

\begin{tabular}{ccccccc}
\hline Sample & $\begin{array}{c}\text { Pinhole } \\
\text { (Airy unit) }\end{array}$ & $\begin{array}{c}\text { Spatial XY } \\
\text { resolution }(\boldsymbol{\mu m})\end{array}$ & $\begin{array}{c}\text { Spatial Z } \\
\text { resolution }(\boldsymbol{\mu m})\end{array}$ & Scan format & Zoom & Voxel size $\left(\mathbf{X} \times \mathbf{Y} \times \mathbf{Z} \boldsymbol{\mu \mathbf { m } ^ { 3 } )}\right.$ \\
\hline $\begin{array}{c}\text { Fly ash (hand- } \\
\text { mixed) }\end{array}$ & 0.3 & 0.156 & 0.534 & $2048 \times 2048$ & $1.8 \times$ & $0.105 \times 0.105 \times 0.1$ \\
$\begin{array}{c}\text { Fly ash (vacuum- } \\
\text { impregnated) }\end{array}$ & 0.3 & 0.156 & 0.534 & $1024 \times 1024$ & $1.46 \times$ & $0.26 \times 0.26 \times 0.25$ \\
$\begin{array}{c}\text { Cement paste } \\
\text { Concrete }\end{array}$ & 0.3 & 0.156 & 0.534 & $2048 \times 2048$ & $1.8 \times$ & $0.105 \times 0.105 \times 0.1$ \\
\hline
\end{tabular}




\section{Results}

\subsection{Reconstructed image volumes}

The reconstructed image volumes for the fly ash (hand-mixed and vacuum-impregnated), cement paste and concrete samples are shown in orthogonal views in Fig. 6 (A), (C), (E) and (G), respectively. All images were converted to grey scale and inverted to highlight the features of interest. Dark areas are the fluorescent epoxy-filled void spaces whereas bright areas correspond to solids. A median filter of $1 \times 1 \times 1$ was applied to the images to remove noise.

The reconstructed fly ash images of both the hand-mixed and vacuum-impregnated samples (Fig. 6 (A) and 6 (C)) shows that most of the fly ash particles are spherical. Some of the particles have heterogeneous texture or have an irregular shape (cenospheres). It is also clearly visible that the vacuum-impregnated sample contained more densely packed fly ash particles. The observable particle size ranges between $3 \mu \mathrm{m}$ to tens of microns. These features observed on the reconstructed image volume correspond well to those seen in 2D imaging such as backscattered electron microscopy. In the orthogonal views (XZ \& YZ), the spherical shape of the reconstructed fly ash particles is clearly evident, indicating the viability of the proposed method. Further results on the reconstructed fly ash particles in the hand-mixed sample are presented in Section 6.3 where comparison is made against the reference image volume and the accuracy of the proposed method is assessed.

The reconstructed cement paste (Fig. 6 (E)) shows many anhydrous cement grains consistent with the low w/c ratio and low degree of hydration of the sample. These cement grains can be easily distinguished because their boundaries are very distinct, examples of these are marked in the figure. The cement grains are surrounded by a heterogeneous matrix of hydration products and capillary pores. The pores appear dark and sizes ranging from around $0.6 \mu \mathrm{m}$ up to $10 \mu \mathrm{m}$ can be observed in the orthogonal views. It is noted that the pores appear somewhat elongated in the orthogonal views; this was expected because of the lower resolution in the Z-direction compared to the XY-direction. Such effect is particularly pronounced when the size of the feature of interest in the microstructure is close to the $\mathrm{Z}$ resolution of LSCM. Given the fact that most capillary pores are sub-micron in size, this effect is therefore more visible in the reconstructed cement paste.

The reconstructed image volume of the concrete sample (Fig. 6 (g)) shows substantial amount of microcracking induced by cyclic freeze-thaw. Bond cracks at the interface between aggregates and cement paste, and matrix cracks that propagate through the cement paste are clearly visible. The smallest microcrack detected was around $0.8 \mu \mathrm{m}$ and the largest observed was about $50 \mu \mathrm{m}$ wide. The 3D morphology of the microcracks is clearly visible in the orthogonal views.

\subsection{Accuracy of reconstructed image volumes}

The details of the reconstructed image volumes are summarised in Table 4. The number of images selected per stack for the hand-mixed fly ash and cement paste samples were 30 images, corresponding to a thickness of $3 \mu \mathrm{m}$. For the concrete sample, the number of images selected per stack was 35 images, corresponding to a larger thickness of $5.2 \mu$ m. This was because the microcracks could be imaged to greater depths without signal degradation. For the vacuumimpregnated fly ash sample, contrast limited adaptive histogram equalisation ${ }^{\dagger}$ (Zuiderveld, 1994) was applied to the images prior to stitching to enhance the local contrast between the epoxy and fly ash particles. This increased the thickness of each image stack that can be selected for stitching. The number of images selected per stack was 30 images, corresponding to a thickness of $7.5 \mu \mathrm{m}$. However, it is noted that contrast limited adaptive histogram equalisation is not effective for increasing the stack thicknesses of cement paste and concrete samples because the microstructures are less distinct at greater depths.

For all samples, the ratio between the average $\Delta \mathrm{Z}$ and the selected stack thickness was no more than 0.74 (see Fig. 4). In other words, the average overlapping region between stacks for each sample was at least 26\%. Although both fly ash samples were subjected to the same grinding time, the average $\Delta \mathrm{Z}$ for the vacuum-impregnated sample was slightly higher than that for the hand-mixed sample. This was expected because the vacuum-impregnated sample was softer due to the presence of densely packed fly ash particles.

The average correlation coefficients $(\mathrm{R})$ for the hand-mixed fly ash sample and concrete were $>0.9$ whereas for the vacuum-impregnated fly ash sample and cement paste, the average $\mathrm{R}$ values were slightly below 0.9 . This shows that the overlapping regions between stacks are nearly identical, highlighting the accuracy of the serial sectioning, stitching and reconstruction. The slightly higher correlation coefficients for the fly ash and concrete samples suggest that accuracy is improved when the stitching is carried out on images containing many large and well-defined features.

Table 4: Summary of reconstruction results.

\footnotetext{
${ }^{\dagger}$ Contrast limited adaptive histogram equalisation splits an image into tiles and equalises the histogram of each tile. Contrast limit is applied to prevent amplification of noise and bilinear interpolation is applied to remove artificial boundaries between tiles.
} 


\begin{tabular}{|c|c|c|c|c|c|c|c|c|c|c|}
\hline \multirow{2}{*}{ Sample } & \multirow{2}{*}{$\begin{array}{l}\text { No. of } \\
\text { z- } \\
\text { stacks }\end{array}$} & \multirow{2}{*}{$\begin{array}{c}\text { No. of } \\
\text { images } \\
\text { selected } \\
\text { per stack }\end{array}$} & \multirow{2}{*}{$\begin{array}{c}\text { Thickness of } \\
\text { each stack } \\
(\mu \mathrm{m})\end{array}$} & \multicolumn{2}{|c|}{$\Delta \mathrm{Z}(\boldsymbol{\mu m})$} & \multicolumn{2}{|c|}{$\begin{array}{l}\text { Overlapping } \\
\text { region (\%) }\end{array}$} & \multicolumn{2}{|c|}{ R (-) } & \multirow{2}{*}{$\begin{array}{c}\text { Reconstructed } \\
\text { image } \\
\text { thickness } \\
(\mu \mathrm{m})\end{array}$} \\
\hline & & & & Average & $\begin{array}{l}\text { Std. } \\
\text { dev. }\end{array}$ & Average & $\begin{array}{l}\text { Std. } \\
\text { dev. }\end{array}$ & Average & $\begin{array}{l}\text { Std. } \\
\text { dev. }\end{array}$ & \\
\hline $\begin{array}{l}\text { Fly ash } \\
\text { (hand- } \\
\text { mixed) }\end{array}$ & 8 & 30 & 3.0 & 1.771 & 0.467 & 41.0 & 15.6 & 0.911 & 0.035 & 15.54 \\
\hline $\begin{array}{c}\text { Fly ash } \\
\text { (vacuum- } \\
\text { impregnated) }\end{array}$ & 18 & 30 & 7.5 & 2.664 & 0.993 & 64.5 & 13.2 & 0.887 & 0.028 & 53.09 \\
\hline $\begin{array}{l}\text { Cement } \\
\text { paste }\end{array}$ & 45 & 30 & 3.0 & 2.223 & 0.528 & 25.9 & 17.6 & 0.874 & 0.024 & 100.95 \\
\hline Concrete & 50 & 35 & 5.2 & 3.296 & 1.228 & 36.6 & 23.6 & 0.922 & 0.040 & 166.93 \\
\hline
\end{tabular}

Fig. 6 (B), (D), (F) and (H) show plots of the computed $\Delta \mathrm{Z}$ and $\mathrm{R}$ versus the cumulative reconstructed image thickness for the fly ash (hand-mixed and vacuum-impregnated), cement paste and concrete samples, respectively. The reconstructed image thicknesses reported here and in Table 4 have been corrected to account for axial distortion caused by mismatch of refractive indices between the immersion oil and epoxy resin using Eq. (3).

Hypothetically, the relationship between $\Delta \mathrm{Z}, \mathrm{R}$ and the sectioning process is as follows: $\Delta \mathrm{Z}$ (equivalent to the thickness of material removed) is dependent on grit size, time and pressure applied during the grinding process. A larger $\Delta \mathrm{Z}$ would result if a thicker layer is removed under a larger grit size, higher grinding pressure or a longer grinding time. Consequently, the overlapping region between stacks would reduce and become less correlated (R). Although we attempted to use the sectioning process described in Section 5.2 throughout this study, the values of $\Delta \mathrm{Z}$ and $\mathrm{R}$ varied slightly between consecutive stacks for all samples. This could be due to the fact that the serial sectioning process was semi-automated and that there was variation in the sample hardness. However, the minimum $\mathrm{R}$ values in all cases were no less than 0.8 , meaning that all consecutive stacks contained overlapping regions that correlated fairly well. It was noted that for all samples, larger $\Delta \mathrm{Z}$ tended to give poorer $\mathrm{R}$, but this relationship was not absolute (see Fig. 7). While the method can be inconsistent, it is worth pointing out that it is not necessary to have uniform thickness of materials removed each time so long as all z-stacks have overlapping regions for stitching.

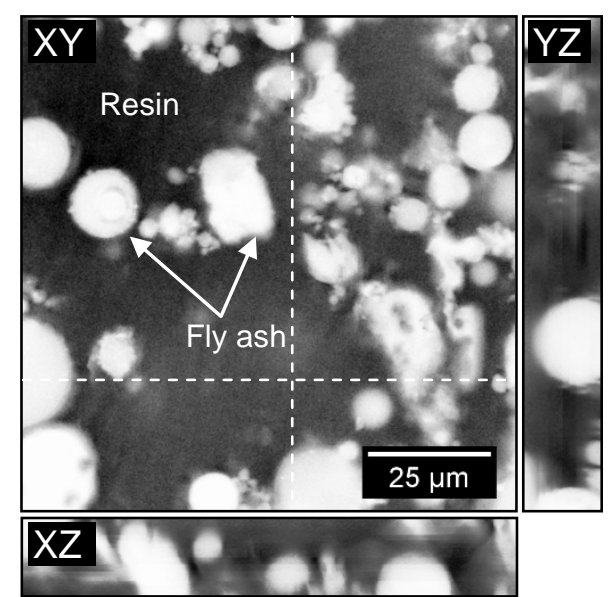

(a)

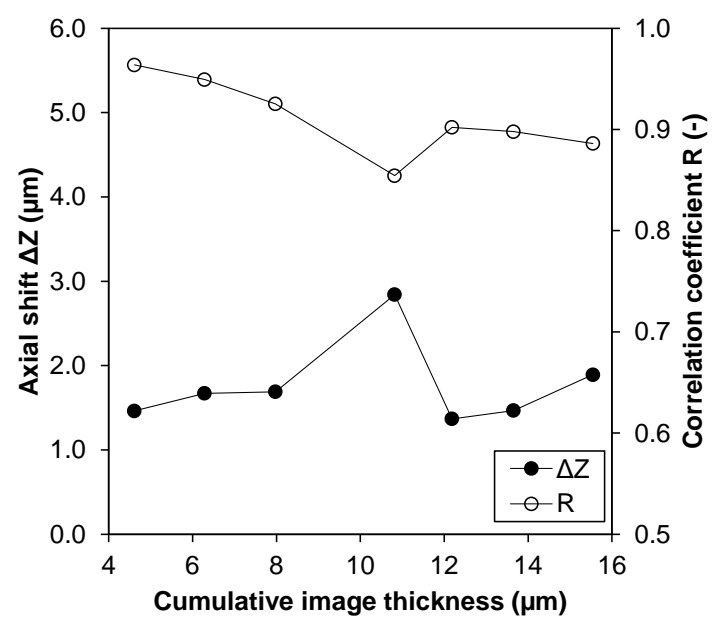

(b) 


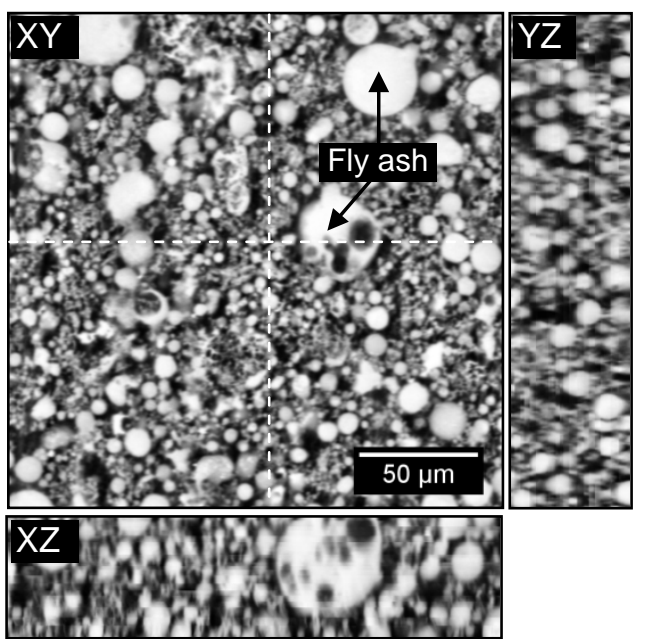

(c)
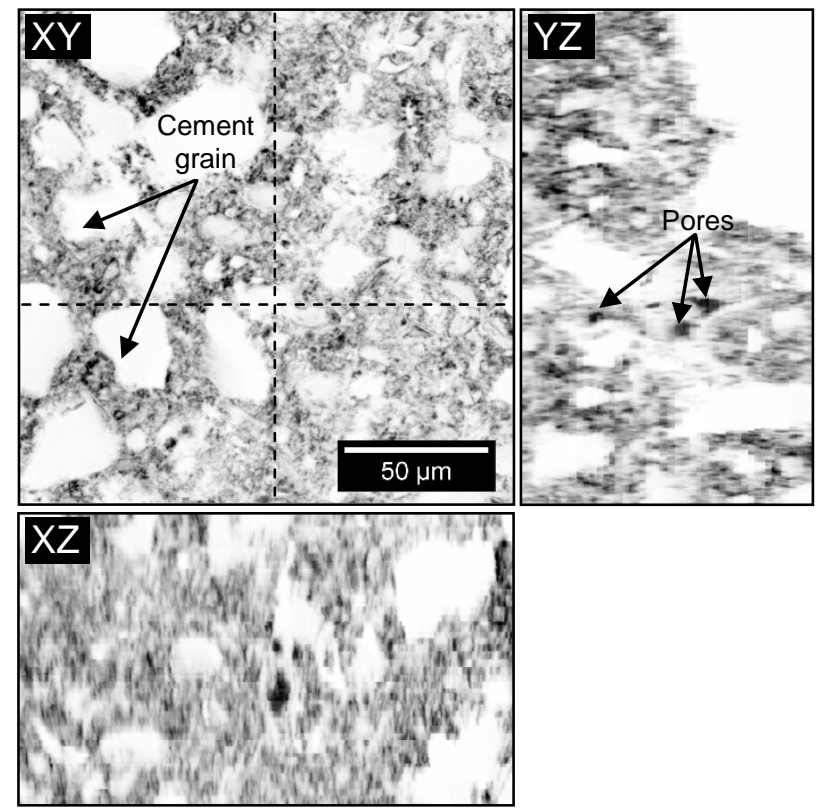

(e)
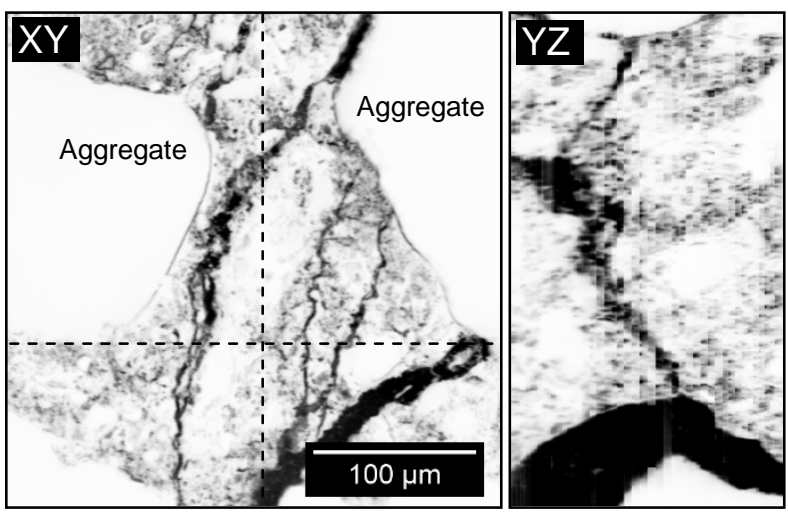

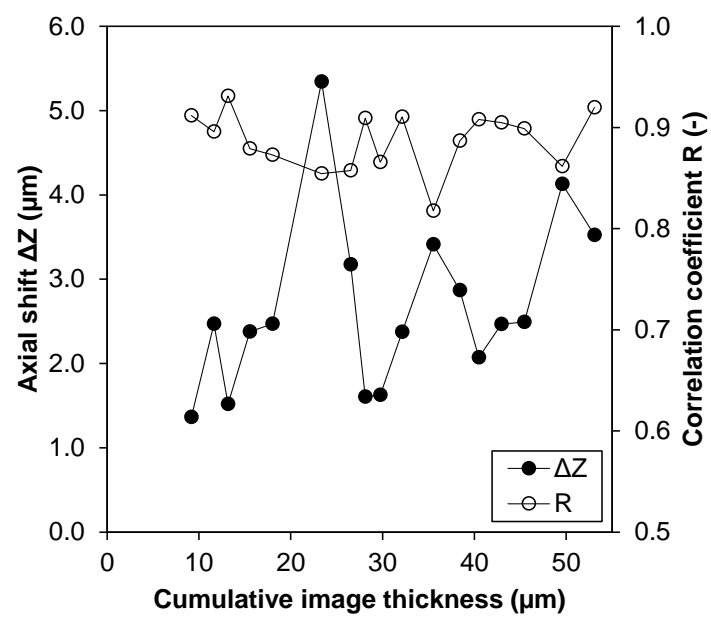

(d)

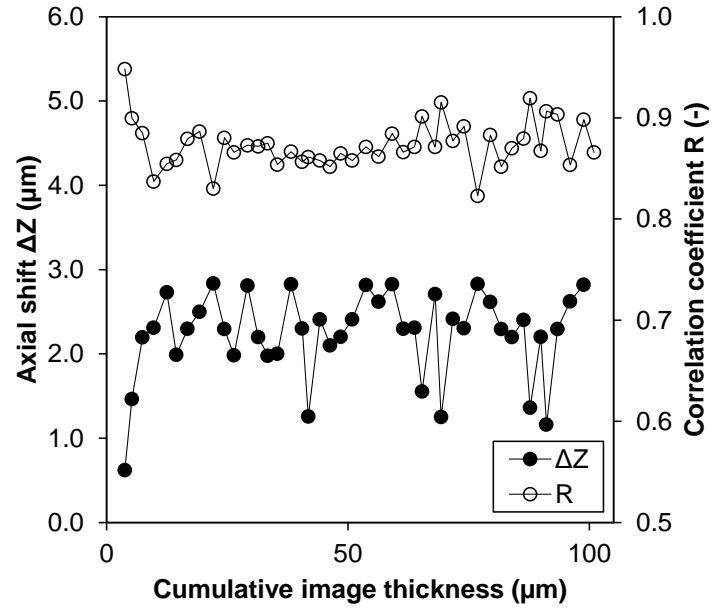

(f)

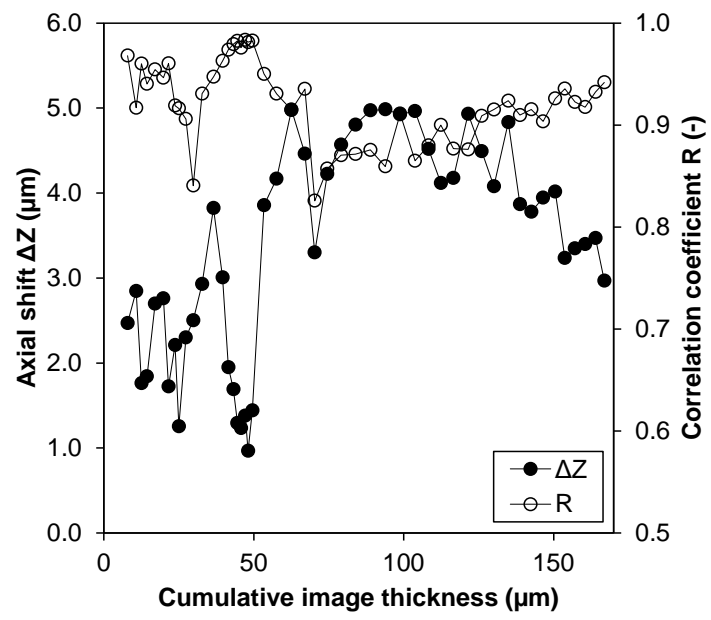

(h) 
Fig. 6. Orthogonal views of reconstructed image volumes and their respective plots of axial shift and correlation coefficient as a function of cumulative image thickness: (A-B) hand-mixed fly ash sample, (C-D) vacuumimpregnated fly ash sample, (E-F) cement paste and (G-H) concrete.

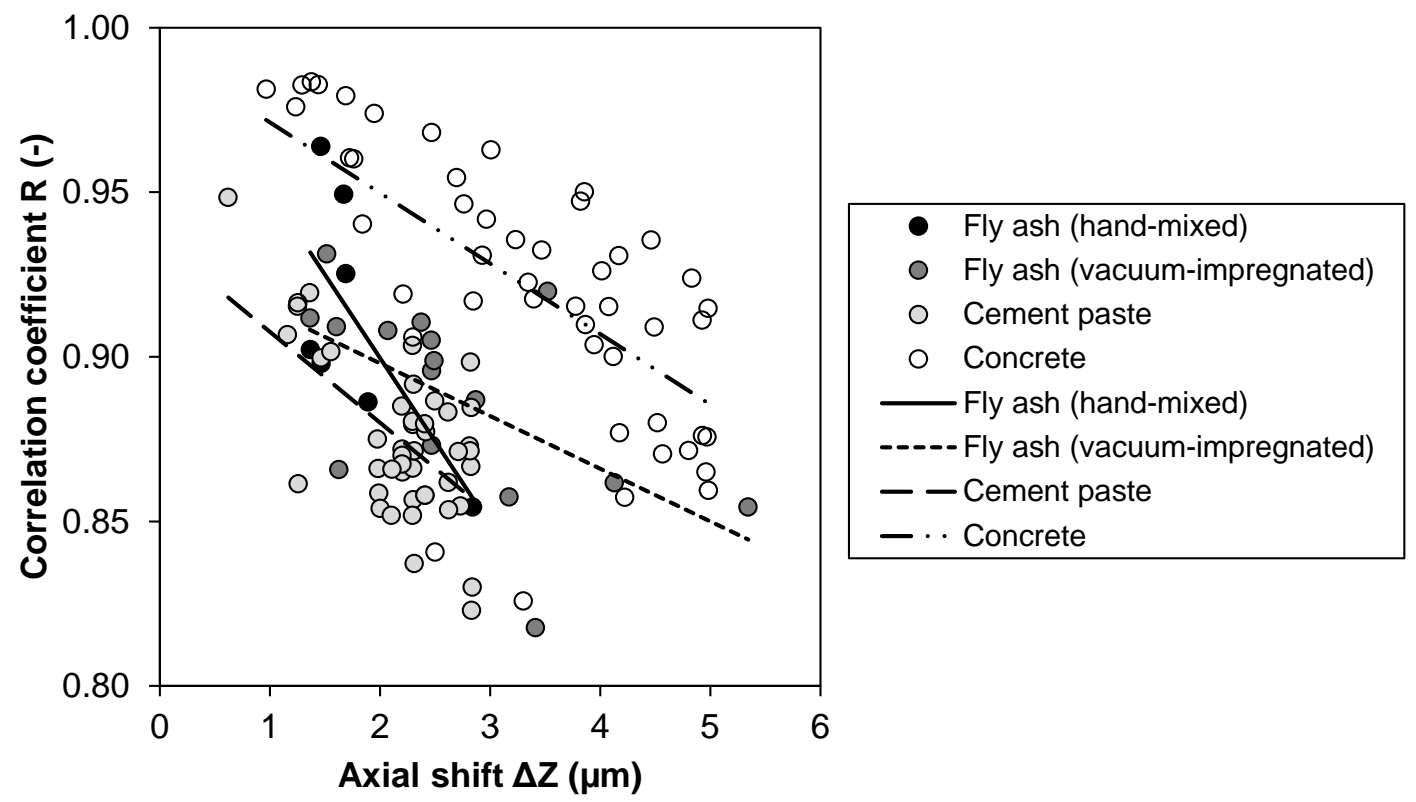

Fig. 7. Plot showing $R$ versus $\Delta \mathrm{Z}$ for all samples.

\subsection{Comparison between reconstructed and reference image volumes of fly ash particles}

As stated in Section 5.3, a reference image volume of approximately $15 \mu \mathrm{m}$ thick was acquired from the hand-mixed fly ash sample prior to the start of the sectioning process for comparison with the reconstructed image volume. Fig. 8 shows area-matching cropped regions from the XY plane of the reference and reconstructed image at increasing depths from the sample surface. It is evident that the quality of the reference image degrades significantly with increasing depth. The fly ash particles in the reference image appear blurry from $4.3 \mu \mathrm{m}$ beneath the surface and gradually become unresolvable due to scattering of the laser and fluorescence signal by overlying particles. However, the quality of the reconstructed image remains consistent and the fly ash particles are resolvable throughout the imaging depth. Images from deeper planes were less grainy compared to those in the reference image volume.
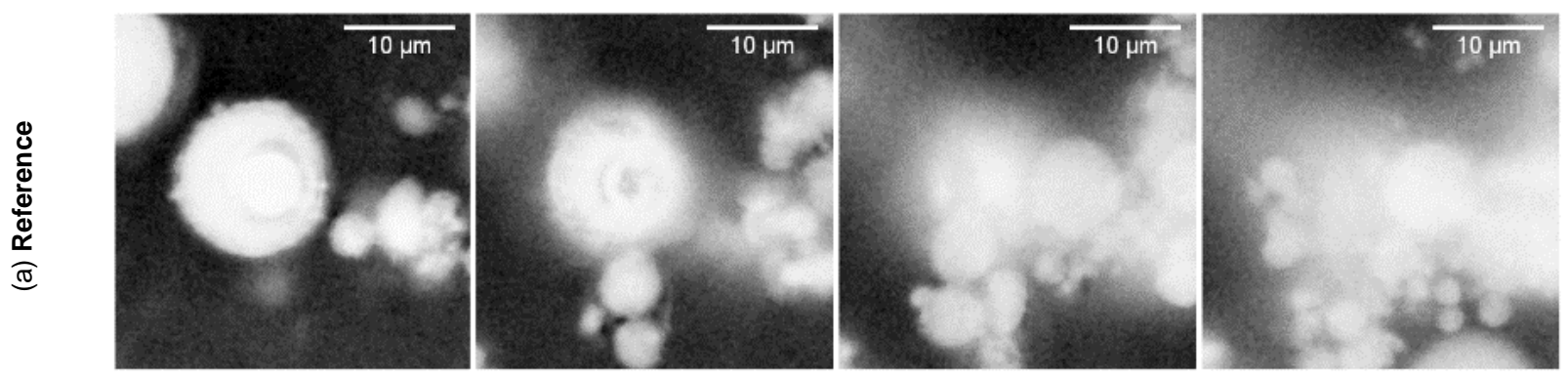

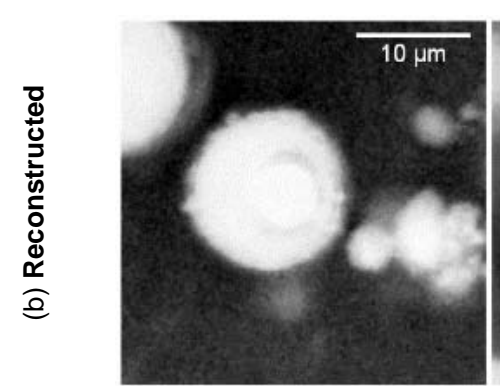

Depth:

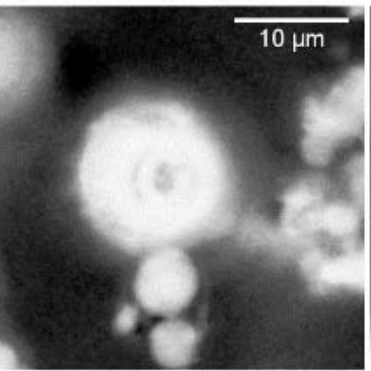

$4.3 \mu \mathrm{m}$

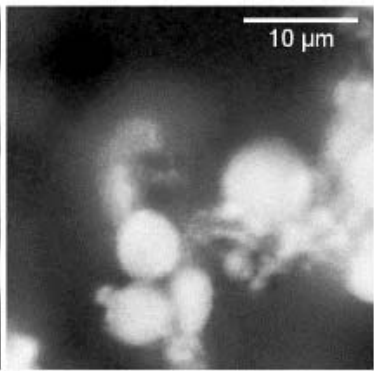

$8.6 \mu \mathrm{m}$

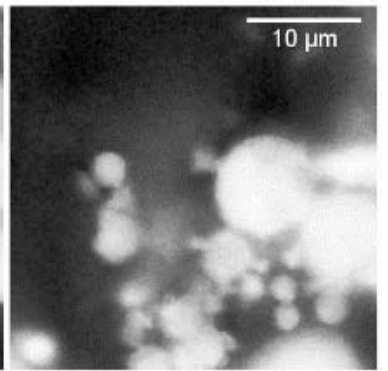

$12.9 \mu \mathrm{m}$ 
Fig. 8. Comparison of area-matching regions (XY-plane) from the reference and reconstructed image of the hand-mixed fly ash sample at increasing depths from the surface showing the accuracy and improved resolution of the reconstructed image.

Fig. 9 compares the reconstructed fly ash particles with their respective originals that could be imaged entirely in the reference image. All images shown are either in the XZ or YZ-plane. The reconstructed fly ash particles are visually identical in terms of shape and size to their originals. Quantitative assessment was carried out by comparing the area, perimeter, Feret's diameter (i.e. the longest distance between any two points along the boundary), circle-equivalent diameter (derived from area) and circularity $\left(=4 \pi^{*}\right.$ area/perimeter $\left.{ }^{2}\right)$ of the reconstructed particles to their originals. The measurements were done in Fiji using the semi-automated Wand (tracing) tool to delineate particle boundaries. This process was repeated thirty times for each particle to obtain an average value for each parameter. The results are plotted in Fig. 10, where error bars show 95\% confidence interval for each data point, calculated using Student's t-distribution. A good agreement can be observed. The average absolute difference (and maximum difference) for area, perimeter, Feret's diameter, circle-equivalent diameter and circularity are 1.75\% (3.06\%), 1.06\% (-1.86\%), 2.46\% (4.74\%), 0.90\% $(1.52 \%)$ and $1.53 \%$ (3.11\%), respectively. There are no systematic positive or negative differences in the measured values except for circularity. Most of the reconstructed particles have slightly higher circularity than their originals. One possible explanation is that the shape of the particles is more defined in the reconstructed images. The calculation of circularity is also rather sensitive to the input values of area and perimeter. Overall, the results show that the serial sectioning and reconstruction process did not distort the reconstructed image.
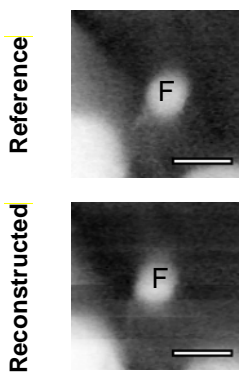

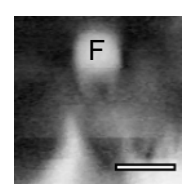

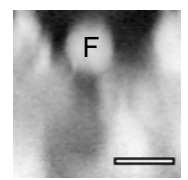
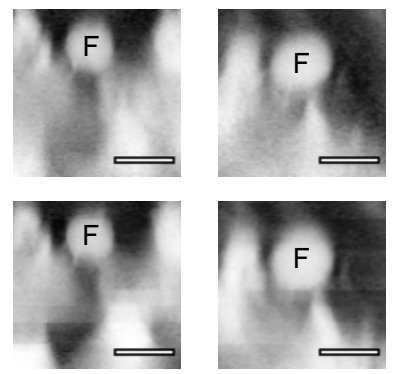
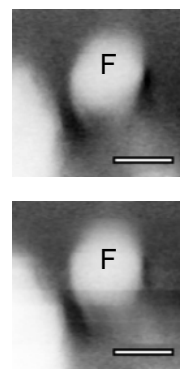
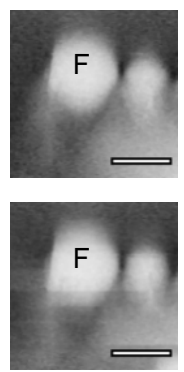
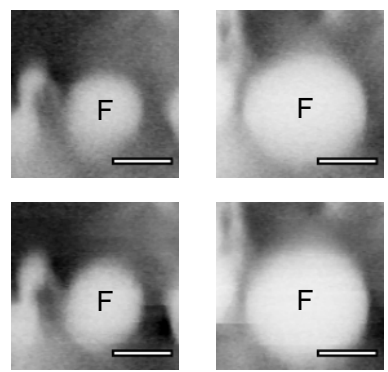

Fig. 9. Comparison of fly ash particles (marked ' $F$ ') obtained from the reference and reconstructed image. These particles are selected from within a depth of $10 \mu \mathrm{m}$ from the surface. Images shown are either in the $\mathrm{XZ}$ or $\mathrm{YZ}$ view. All scale bars are $5 \mu \mathrm{m}$.

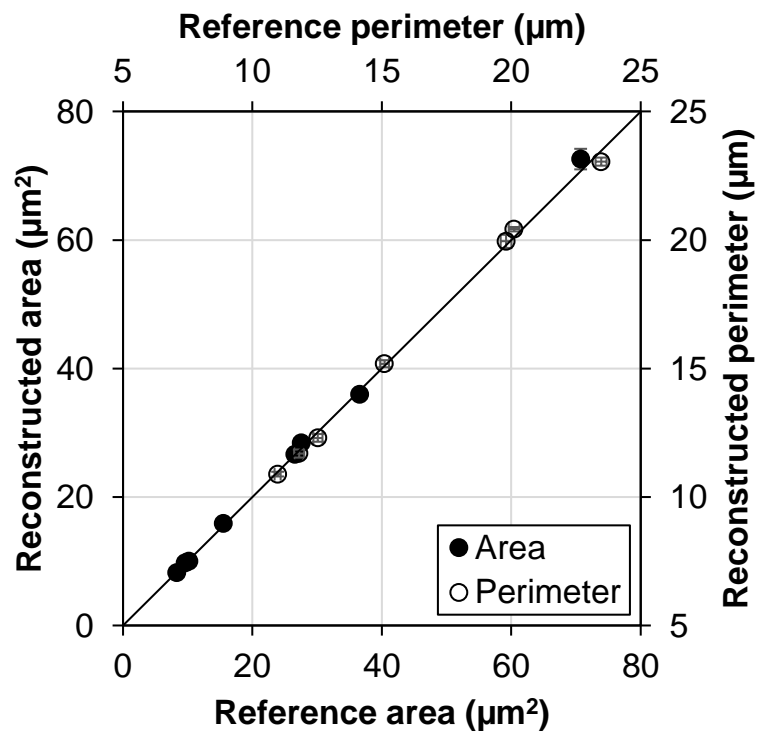

(a)

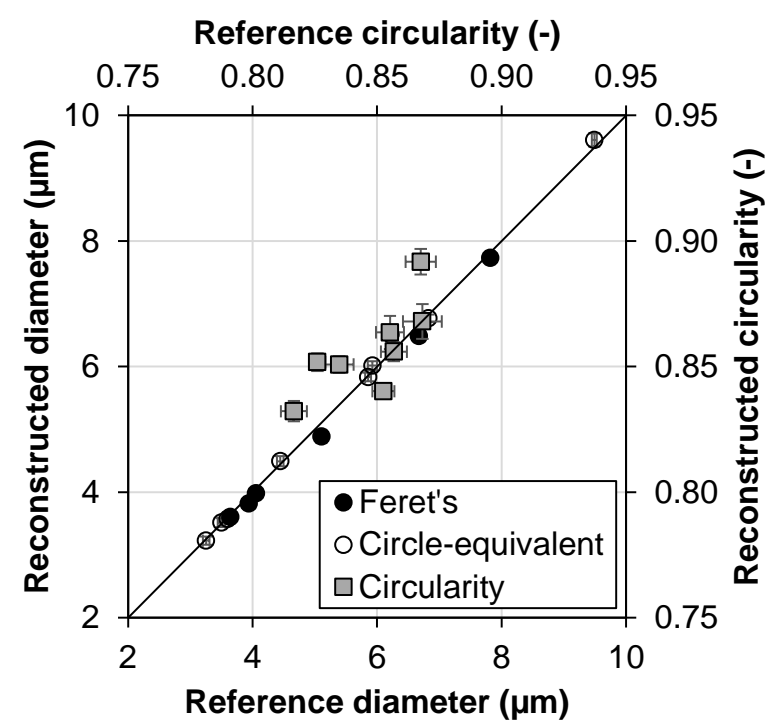

(b)

Fig. 10. Quantitative comparison of reconstructed fly ash particles with their originals in terms of (A) area and perimeter, and (B) Feret's diameter, circle-equivalent diameter and circularity.

\subsection{Applications for cement-based materials}

For cement-based materials, LSCM images only void spaces that contain fluorescent epoxy. Voids that are isolated are not 'seen' as these would not be impregnated with the fluorescent epoxy. Therefore, the technique lends itself very well for the purpose of characterising interconnected pores and cracks that influence mass transport properties. Fig. 11 shows 
a 3D rendering of the cement paste reconstructed in this study. The interconnected pores are segmented by grey level thresholding and surface rendered. The pores are then skeletonised to produce a network of single-voxel pathways along the geometric centre of the pore channels. Thus, the complex pore structure is reduced to a skeletal network that retains the original topology, allowing the development of algorithms to quantify connectivity, orientation and tortuosity. The number of pores connected at pore chambers (nodes) gives the distribution of co-ordination number. Pore throats can also be identified through a search of minima in the hydraulic radius of individual channels to measure constriction factors.

Cement-based materials contain micro-cracks induced by shrinkage, thermal effects, deleterious mechanisms or mechanical loading. Fig. 12 shows a 3D rendering of the concrete sample examined in this study and the extracted microcracks caused by freeze-thaw action. It is expected that microcracks have a significant effect on transport properties, but very few studies have been carried out to thoroughly characterise the microcracks or correlate their characteristics to transport. More importantly, no attempts have been made to isolate the influence of microcracks from other factors such as pore structure and degree of saturation on transport properties. This is partly because none of the existing techniques is able to image a representative volume of concrete in $3 \mathrm{D}$ at sufficiently high resolution to capture the microcracks. The proposed method can facilitate this to some extent.

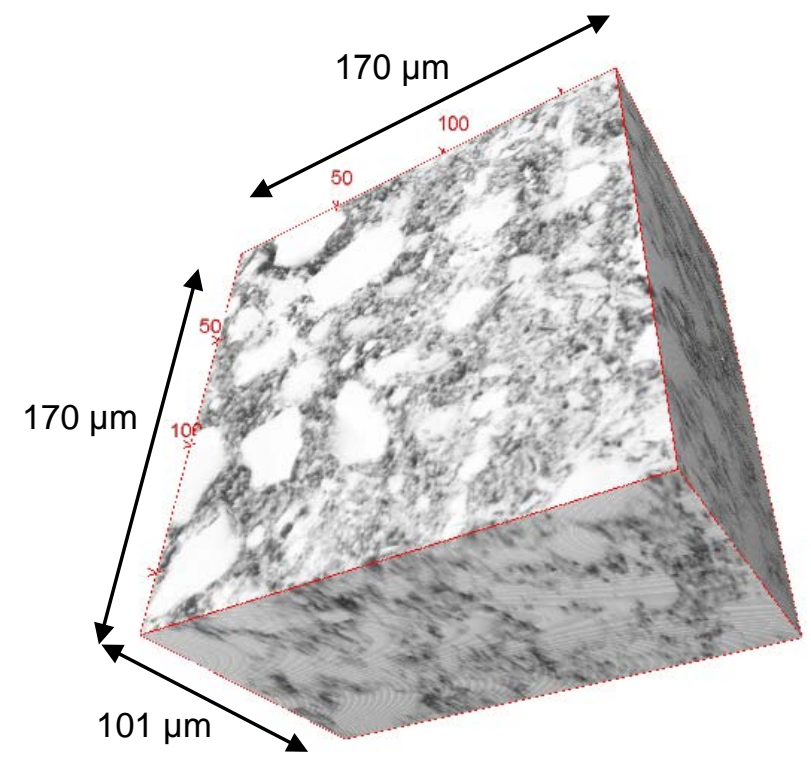

(a)

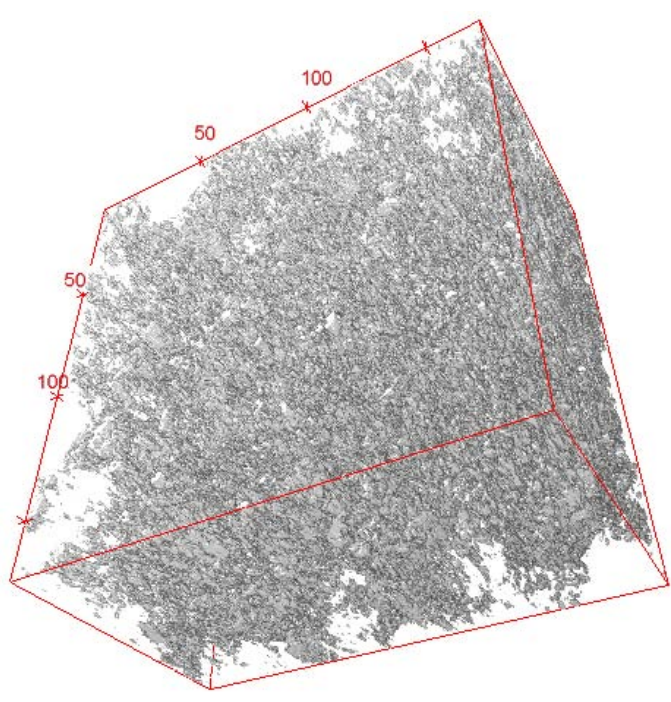

(b)

Fig. 11. 3D rendering of the $(A)$ reconstructed cement paste and $(B)$ extracted pore network.

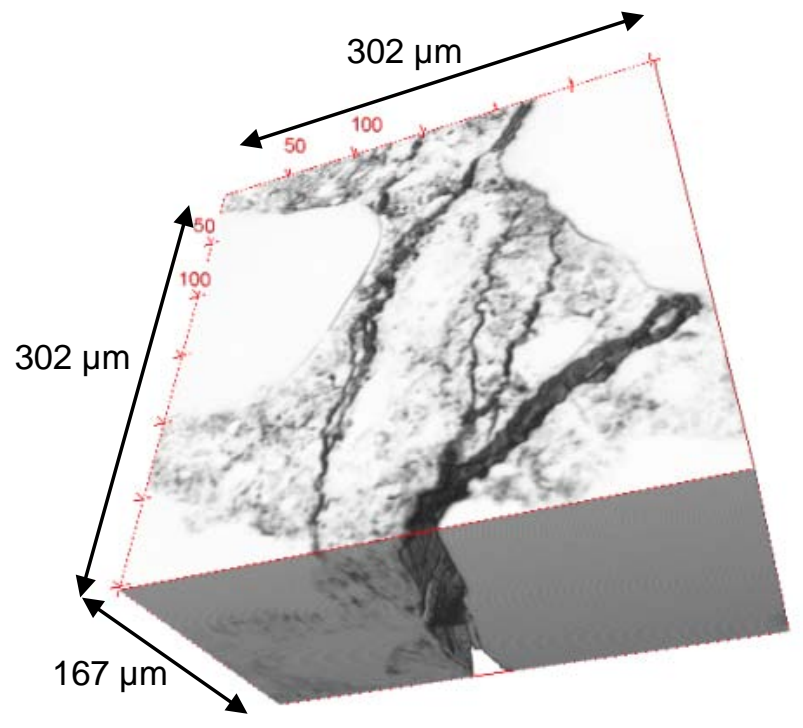

(a)

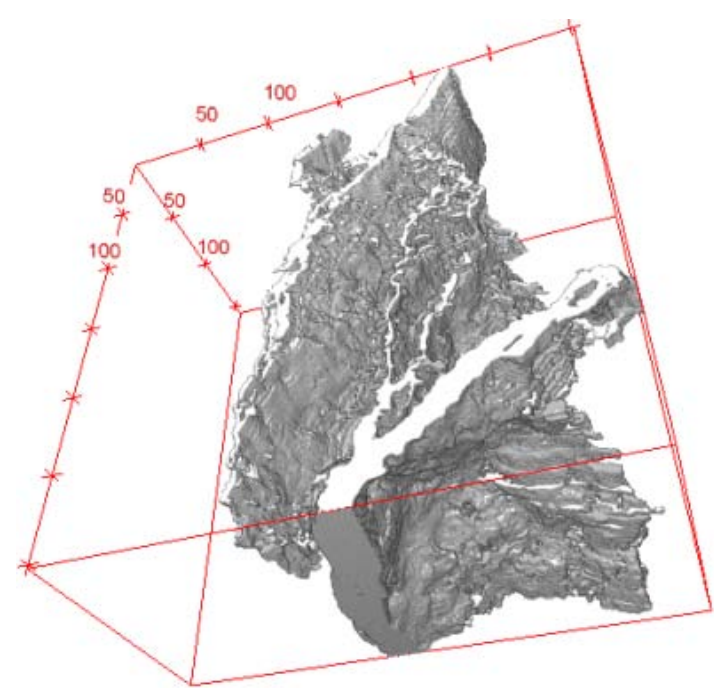

(b)

Fig. 12. 3D rendering of the (A) reconstructed concrete and (B) extracted microcracks.

Another application of the proposed method is characterising 3D particle shape of cements, supplementary cementitious materials and fillers. This is illustrated on a small volume of Portland cement particles embedded in epoxy resin in Fig. 
13. The particle shape of cementitious materials has important influences on the rheology and hydration kinetics. Therefore, the ability to characterise their 3D particle shapes would help enhance our understanding and our ability to model these effects. Existing imaging techniques such as FIB-nt and synchrotron $\mu \mathrm{CT}$ are able to cover only particles in the ranges between 0.4 to $2.0 \mu \mathrm{m}$ and 20 to $60 \mu \mathrm{m}$, respectively (Holzer et al., 2010). The proposed method can be used to image a wider range of particle size that bridges the gap between these two techniques. For instance, with a 40× immersion oil objective lens, the smallest particle that can be characterised is about $3 \mu \mathrm{m}$ (10 voxels/direction is normally used for shape analysis (Garboczi \& Bullard, 2004); by setting the voxel size to half the Z resolution, 0.534 $\mu \mathrm{m}$ ( 0.3 Airy unit), 10 voxels is equal to 5 times the $\mathrm{Z}$ resolution $\approx 3 \mu \mathrm{m}$ ) and the field of view can be extended to more than $700 \times 700 \mu \mathrm{m}^{2}$ in the XY plane and infinitely in the $\mathrm{Z}$ direction to cover a statistically significant number of particles.

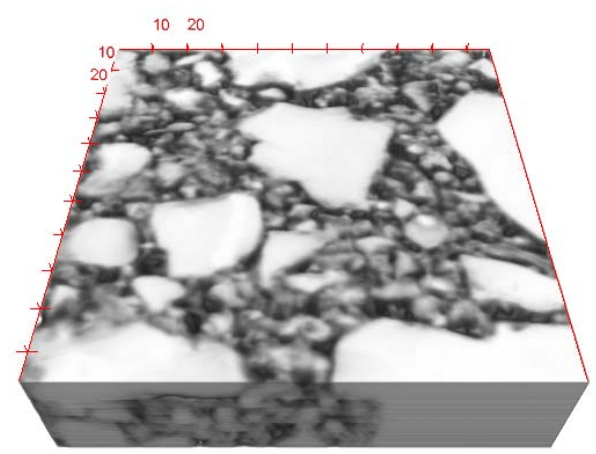

(a)

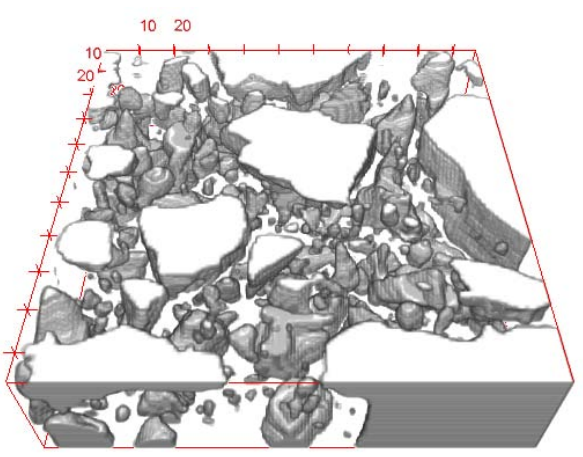

(b)

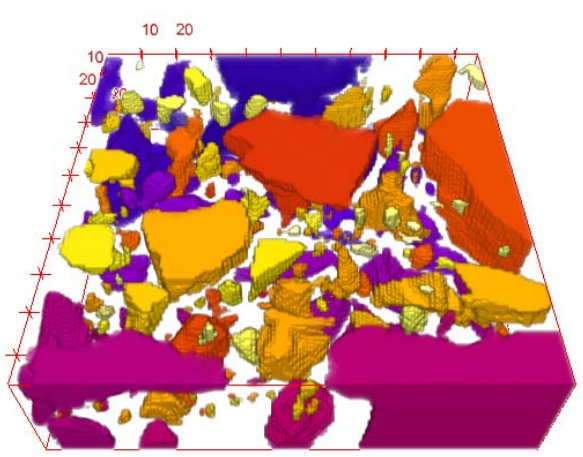

(c)

Fig. 13. 3D rendering of (a) reconstructed (b) segmented and (c) colour-coded Portland cement particles for further analysis.

\section{Discussion}

Any imaging technique is inherently limited in the size of the largest sample that it can image and its ability to resolve the smallest features present in it. The proposed method has been shown to be able to reconstruct thick volumes of cement-based materials from series of fluorescence LSCM z-stacks at sub-micron resolutions with good accuracy. The method can also be extended by stitching in the XY plane to further increase the field of view and hence the overall image volume without loss of resolution. Fig. 14 shows an example of this where the concrete sample was imaged as four $(2 \times 2)$ overlapping mosaic tiles and subsequently reconstructed. The overlapping regions between the mosaic tiles were around $20 \%$. Here, several aggregates are fully captured and the propagation of the microcracks can be better defined compared to that shown in Fig. $6(\mathrm{G})$.

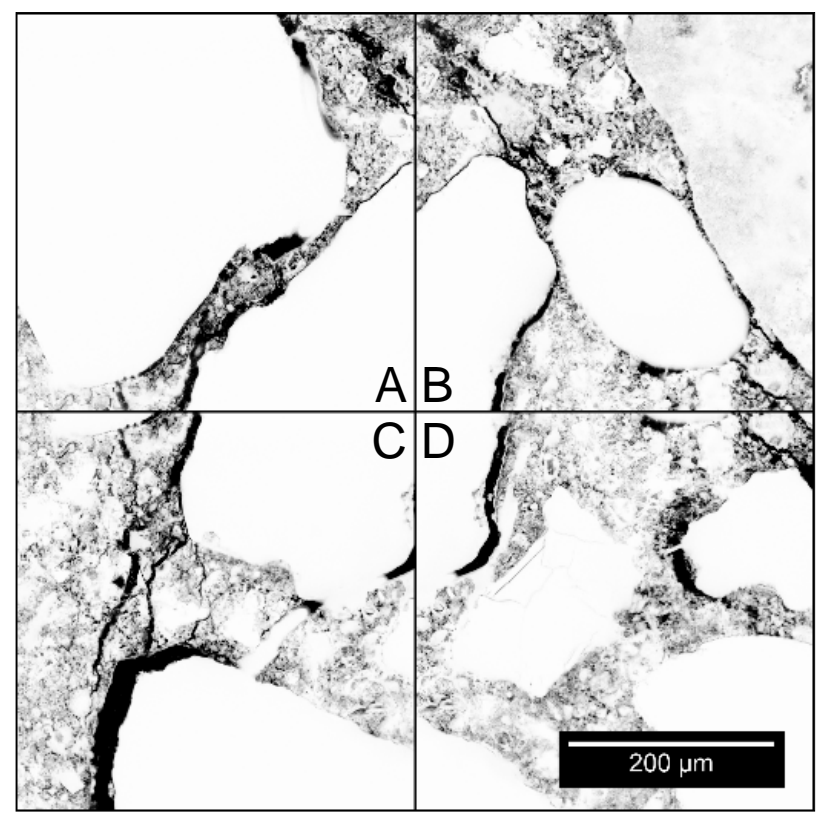

(a)

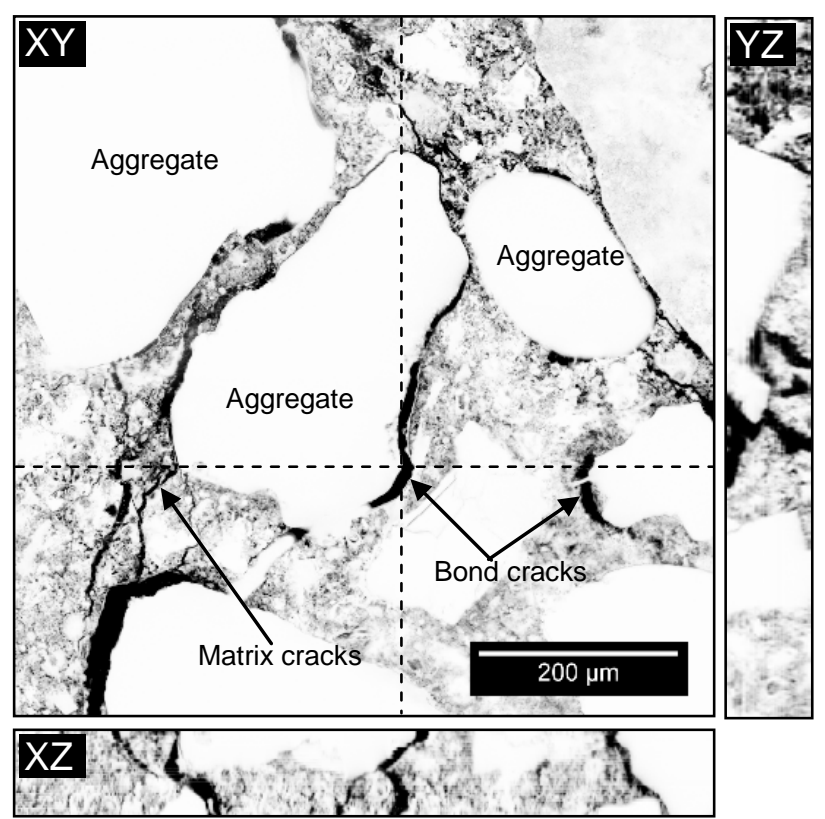

(b) 
Fig. 141. Extending the image volume by stitching in the XY plane. (A) Four individual mosaic tiles (1, 2, 3 and 4) each with an image area of $387 \times 387 \mathrm{\mu m}^{2}(\mathrm{~B})$ Orthogonal views of the image volume reconstructed from the mosaics in (A), total area in $\mathrm{XY}$ is $700 \times 700 \mathrm{\mu m}^{2}$.

Theoretically, the image volumes can be extended infinitely in both the XY and Z planes. However, in practice there are several factors that could limit this. For example, it is difficult to ensure that the sample is perfectly flat and equal thickness throughout, particularly for a large sample. Therefore, it may not be possible to capture mosaic tiles that cover the entire sample at the same focal plane. The sectioning method (i.e. mechanical grinding) also causes the edges of the sample to be worn more easily than the centre. The height of the stage can be adjusted to accommodate this to a certain degree, but reconstruction could become problematic if there is a large discrepancy in the focal depth of the images in each tile.

The depth of sample that can be imaged is limited by the impregnation depth of the fluorescent resin since imaging is done in the fluorescence mode. The impregnation depth is dependent on the method of impregnation, viscosity of resin and the pore structure of the sample. In this study, the impregnation depth of fluorescent resin was $>100 \mu \mathrm{m}$ and $>3$ $\mathrm{cm}$ for the cement paste and concrete sample respectively. However, the sample can be re-impregnated with resin if one wishes to image thicker sections. This issue is not relevant to the hand-mixed fly ash sample as it was directly embedded in fluorescent epoxy resin.

LSCM images can suffer from uneven illumination and shadowing effects in the XY plane, especially when a large area is imaged and the sample is not perfectly flat and horizontal. In such instance, the image can be corrected prior to alignment by means of pseudo flat-field correction. This is carried out by dividing the raw image by a flat-field image generated by mean-filtering the raw image using a large kernel size) (see Fig. 15.). Uneven brightness may also occur along the depth of reconstructed image volumes due to factors such as varying imaging conditions after each cycle of sectioning, inherent inconsistencies or heterogeneities in the sample and/or photo bleaching of the fluorophores due to pro-longed exposure to laser. This can be rectified using histogram matching (Castleman, 1996, Camp et al., 1997) that matches the histograms of successive image slices to that of the first image of the reconstructed image volume to give uniform intensity along the depth.

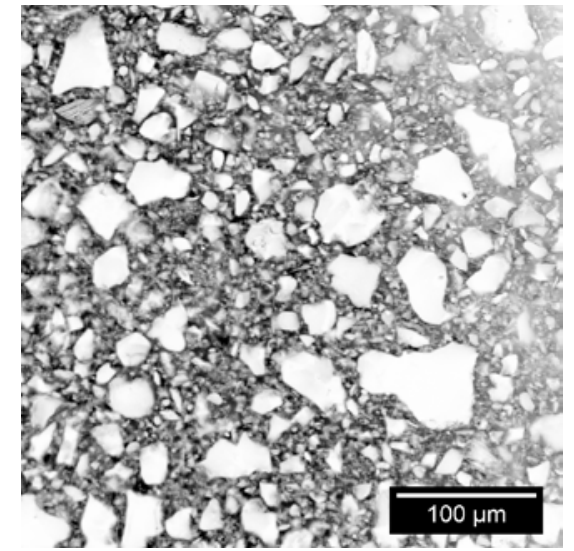

(a)

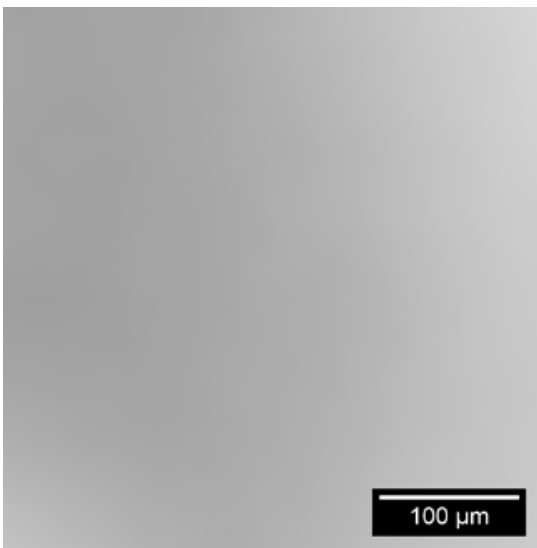

(b)

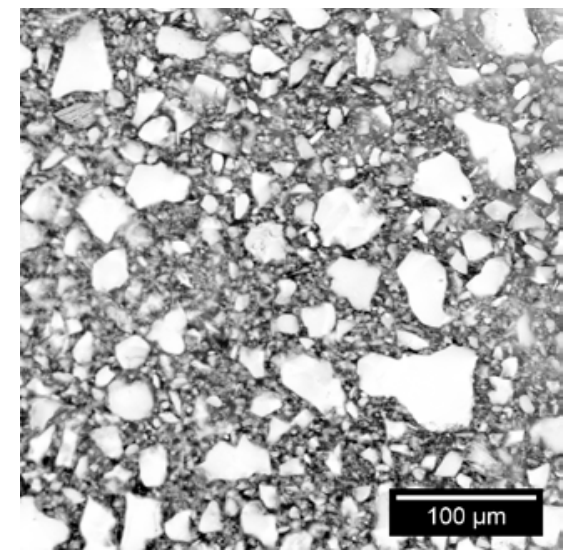

(c)

Fig. 15. Correction of uneven brightness in the XY plane of a LSCM image: (A) raw image (B) flat field image generated by mean filtering (A) with a kernel size of 350 pixels, and (C) corrected image.

Unlike conventional serial sectioning methods such as microtome and cryostat sectioning, our method requires each zstack to have overlapping regions with neighbouring stacks for stitching. This avoids the risk of information lost between stacks. The resolution of LSCM is highly dependent on the objective used. High powered objectives with high numerical apertures (e.g. 40×, 63× and 100×) are able to achieve XY and Z resolutions of down to $\leq 0.2 \mu \mathrm{m}$ and $\leq 0.5$ $\mu \mathrm{m}$, respectively. These can be possibly further improved by means of deconvolution (Pawley, 1995) to restore the images based on the knowledge of the point-spread function (PSF) that can either be measured experimentally from a microsphere or generated theoretically based on the configurations of the imaging system.

The main drawback of the proposed method is that depending on the image volume and resolution required, the process of serial sectioning, imaging and reconstruction may be laborious and time consuming. For example, the average time required to serial section and image one $2.5 \mu \mathrm{m}$ thick stack at a $0.534 \mu \mathrm{m} \mathrm{Z}$ resolution from a cement paste sample is approximately 45 minutes using our current facilities. However, we envisage that this can be improved by automating the process. Serial sectioning is intrusive, meaning that the sample cannot be revisited once sectioned. Scratches may also be introduced during the grinding process, but this problem can be avoided if one selects images well below the surface for reconstruction. Another disadvantage of LSCM is that it cannot distinguish solid phases of different compositions. One way to circumvent this is to carry out parallel imaging where required using SEM with energydispersive X-ray spectroscopy to identify the solid phases of interest. 
The proposed method is suitable for dense materials containing pores in the range of sub-micron to tens of microns. Other materials that can be characterised by the proposed method include permeable rocks that are of importance to the oil industry such as carbonates and limestone (e.g. Ketton and Estaillades) containing highly connected micropores that cannot be captured by X-ray $\mu \mathrm{CT}$ (Blunt et al., 2013). The ability to image these pores in 3D would help improve the understanding and modelling of flow processes of oil and gas through these rocks. Bioceramics that are used in medical applications as bone substitutes are another example. These materials contain micropores of less than10 $\mu \mathrm{m}$ (Dorozhkin, 2010) and the interconnectivity of the pores influences their bioactivity through the transfer/adsorption of nutrients or cell anchorage, leading to the apposition of new bone (Hing et al., 2005).

\section{Conclusion}

This paper presents a new method to image large volumes of non-transparent porous materials at sub-micron resolution in 3D by combining fluorescence LSCM with serial sectioning. The novelty of the method is that the serial sections are imaged to produce consecutive z-stacks containing overlapping regions. This allows stitching based on phase correlation and avoids loss of information between stacks. Furthermore, the resolution of the reconstructed image volume does not degrade with increase in sample size or thickness. The reconstruction process is entirely performed in Fiji. We have used the proposed method to reconstruct fly ash particles, hardened cement paste and concrete with XY and $\mathrm{Z}$ resolutions of down to $\sim 0.2 \mu \mathrm{m}$ and $\sim 0.5 \mu \mathrm{m}$ respectively. The results obtained show the reliability of the method. The proposed method can be used to characterise the 3D shape of cementitious materials and the structure of interconnected pores and microcracks that are important to transport processes. The proposed method fills the critical gap between X-ray $\mu \mathrm{CT}$ and FIB-nt in terms of achievable spatial resolution (sub-micron) and image volume on cement-based materials.

\section{Acknowledgements}

M.H.N. Yio would like to acknowledge the Dixon scholarship provided by the Department of Civil and Environmental Engineering, Imperial College London. M.J. Mac would like to acknowledge the funding from the European Union Seventh Framework Programme (FP7/2007-2013) under grant agreement 264448. We would also like to thank the Facility for Imaging by Light Microscopy (FILM) at Imperial College London for access to their two-photon microscope and Mr Andrew Morris for his assistance in sample preparation.

\section{References}

Bentz, D. P., Mizell, S., Satterfield, S., Devaney, J., George, W., Ketcham, P., Graham, J., Porterfield, J., Quenard, D. \& Vallee, F. (2002) The visible cement data set. Journal of Research of the National Institute of Standards and Technology, 107, 137-148.

Blunt, M. J., Bijeljic, B., Dong, H., Gharbi, O., Iglauer, S., Mostaghimi, P., Paluszny, A. \& Pentland, C. (2013) Porescale imaging and modelling. Advances in Water Resources, 51, 197-216.

Camp, J. J., Hann, C. R., Johnson, D. H., Tarara, J. E. \& Robb, R. A. (1997) Three-dimensional reconstruction of aqueous channels in human trabecular meshwork using light microscopy and confocal microscopy. Scanning, 19, 258-263.

Čapek, M., Brůža, P., Janáček, J., Karen, P., Kubínová, L. \& Vagnerová, R. (2009) Volume reconstruction of large tissue specimens from serial physical sections using confocal microscopy and correction of cutting deformations by elastic registration. Microscopy Research and Technique, 72, 110-119.

Castleman, K. R. (1996) Digital image processing, Prentice Hall, Englewood Cliffs.

Dauzères, A., Le Bescop, P., Cau-Dit-Coumes, C., Brunet, F., Bourbon, X., Timonen, J., Voutilainen, M., Chomat, L. \& Sardini, P. (2014) On the physico-chemical evolution of low-pH and CEM I cement pastes interacting with Callovo-Oxfordian pore water under its in situ CO2 partial pressure. Cement and Concrete Research, 58, 7688.

Desbois, G., Urai, J. L., PÉRez-Willard, F., Radi, Z., Offern, S., Burkart, I., Kukla, P. A. \& Wollenberg, U. (2013) Argon broad ion beam tomography in a cryogenic scanning electron microscope: a novel tool for the investigation of representative microstructures in sedimentary rocks containing pore fluid. Journal of Microscopy, 249, 215-235. 
Diamond, S. (2000) Mercury porosimetry: an inappropriate method for the measurement of pore size distributions in cement-based materials. Cement and Concrete Research, 30, 1517-1525.

Dorozhkin, S. V. (2010) Bioceramics of calcium orthophosphates. Biomaterials, 31, 1465-1485.

Ficker, T., Martišek, D. \& Jennings, H. M. (2010) Roughness of fracture surfaces and compressive strength of hydrated cement pastes. Cement and Concrete Research, 40, 947-955.

Gallucci, E., Scrivener, K., Groso, A., Stampanoni, M. \& Margaritondo, G. (2007) 3D experimental investigation of the microstructure of cement pastes using synchrotron X-ray microtomography $(\mu \mathrm{CT})$. Cement and Concrete Research, 37, 360-368.

Garboczi, E. J. \& Bullard, J. W. (2004) Shape analysis of a reference cement. Cement and Concrete Research, 34, 1933-1937.

Head, M. K. \& Buenfeld, N. R. (2006) Confocal imaging of porosity in hardened concrete. Cement and Concrete Research, 36, 896-911.

Head, M. K., Wong, H. S. \& Buenfeld, N. R. (2006) Characterisation of 'Hadley’ grains by confocal microscopy. Cement and Concrete Research, 36, 1483-1489.

Head, M. K., Wong, H. S. \& Buenfeld, N. R. (2008) Characterising aggregate surface geometry in thin-sections of mortar and concrete. Cement and Concrete Research, 38, 1227-1231.

Hing, K. A., Annaz, B., Saeed, S., Revell, P. A. \& Buckland, T. (2005) Microporosity enhances bioactivity of synthetic bone graft substitutes. J Mater Sci: Mater Med, 16, 467-475.

Holzer, L., Flatt, R. J., Erdoğan, S. T., Bullard, J. W. \& Garboczi, E. J. (2010) Shape comparison between 0.4-2.0 and 20-60 $\mu \mathrm{m}$ cement particles. Journal of the American Ceramic Society, 93, 1626-1633.

Holzer, L., Gasser, P. H., Kaech, A., Wegmann, M., Zingg, A., Wepf, R. \& Muench, B. (2007) Cryo-FIBnanotomography for quantitative analysis of particle structures in cement suspensions. Journal of Microscopy, 227, 216-228.

Holzer, L., Muench, B., Wegmann, M., Gasser, P. \& Flatt, R. J. (2006) FIB-Nanotomography of particulate systemsPart I: Particle shape and topology of interfaces. Journal of the American Ceramic Society, 89, 2577-2585.

Hrnčír, T., Lopour, F., Zadražil, M., Jiruše, J., Delobbe, A., O Salord, O. \& P Sudraud, P. (2012) Novel plasma FIB/SEM for high speed failure analysis, 3D tomography and other applications. In: The 15th European Microscopy Congress. Manchester Central, United Kingdom.

Hsieh, J. (2003) Computed tomography: principles, design, artifacts, and recent advances, SPIE, Bellingham, Washington.

Kim, K. Y., Yun, T. S., Choo, J., Kang, D. H. \& Shin, H. S. (2012) Determination of air-void parameters of hardened cement-based materials using X-ray computed tomography. Construction and Building Materials, 37, 93-101.

Kurtis, K. E., El-Ashkar, N. H., Collins, C. L. \& Naik, N. N. (2003) Examining cement-based materials by laser scanning confocal microscopy. Cement and Concrete Composites, 25, 695-701.

Lange, D. A., Jennings, H. M. \& Shah, S. P. (1993) Relationship between fracture surface roughness and fracture behavior of cement paste and mortar. Journal of the American Ceramic Society, 76, 589-597.

Lavery, L. L., Gelb, J., Merkle, A. P. \& Steinbach, A. (2014) X-Ray microscopy for hierarchical multi-scale materials. Microscopy Today, 22, 16-21.

Lu, S., Landis, E. N. \& Keane, D. T. (2006) X-ray microtomographic studies of pore structure and permeability in Portland cement concrete. Materials and Structures, 39, 611-620.

Luzzati, F., Fasolo, A. \& Peretto, P. (2011) Combining confocal laser scanning microscopy with serial section reconstruction in the study of adult neurogenesis. Frontiers in Neuroscience, 5, 70.

Mac, M., Desbois, G., Pardo, P. \& Casanova, I. (2012) Ion beam tomography of the nano- and microstructure of cementitious materials. In: 4th International Symposium on Nanotechnology in Construction (NICOM-4). Agios Nikolaos, Crete, Greece.

Mauko, A., Muck, T., Mirtič, B., Mladenovič, A. \& Kreft, M. (2009) Use of confocal laser scanning microscopy (CLSM) for the characterization of porosity in marble. Materials Characterization, 60, 603-609.

Monteiro, P. J. M., Kirchheim, A. P., Chae, S., Fischer, P., MacDowell, A. A., Schaible, E. \& Wenk, H. R. (2009) Characterizing the nano and micro structure of concrete to improve its durability. Cement and Concrete Composites, 31, 577-584. 
Münch, B., Gasser, P., Holzer, L. \& Flatt, R. (2006) FIB-Nanotomography of particulate systems—Part II: Particle recognition and effect of boundary truncation. Journal of the American Ceramic Society, 89, 2586-2595.

Pawley, J. B. (1995) Handbook of biological confocal microscopy, Plenum Press, New York.

Petford, N., Davidson, G. \& Miller, J. (2001) Investigation of the petrophysical properties of a porous sandstone sample using confocal scanning laser microscopy. Petroleum Geoscience, 7, 99-105.

Preibisch, S., Saalfeld, S. \& Tomancak, P. (2009) Globally optimal stitching of tiled 3D microscopic image acquisitions. Bioinformatics, 25, 1463-1465.

Promentilla, M. A. B. \& Sugiyama, T. (2010) X-ray microtomography of mortars exposed to freezing-thawing action. Journal of Advanced Concrete Technology, 8, 97-111.

Provis, J. L., Myers, R. J., White, C. E., Rose, V. \& van Deventer, J. S. J. (2012) X-ray microtomography shows pore structure and tortuosity in alkali-activated binders. Cement and Concrete Research, 42, 855-864.

Provis, J. L., Rose, V., Winarski, R. P. \& van Deventer, J. S. (2011) Hard X-ray nanotomography of amorphous aluminosilicate cements. Scripta Materialia, 65, 316-319.

Rougelot, T., Burlion, N., Bernard, D. \& Skoczylas, F. (2010) About microcracking due to leaching in cementitious composites: X-ray microtomography description and numerical approach. Cement and Concrete Research, 40, 271-283.

Schindelin, J., Arganda-Carreras, I., Frise, E., Kaynig, V., Longair, M., Pietzsch, T., Preibisch, S., Rueden, C., Saalfeld, S. \& Schmid, B. (2012) Fiji: an open-source platform for biological-image analysis. Nature Methods, 9, 676682.

Scrivener, K. L. (2004) Backscattered electron imaging of cementitious microstructures: understanding and quantification. Cement and Concrete Composites, 26, 935-945.

Stutzman, P. (9 May 2014) Email sent to M.H.N. Yio.

Stutzman, P. E. (1990) Serial sectioning of hardened cement paste for scanning electron microscopy. Ceram. Trans., 16, 237-249.

Sugiyama, T., Promentilla, M. A. B., Hitomi, T. \& Takeda, N. (2010) Application of synchrotron microtomography for pore structure characterization of deteriorated cementitious materials due to leaching. Cement and Concrete Research, 40, 1265-1270.

Thevenaz, P., Ruttimann, U. E. \& Unser, M. (1998) A pyramid approach to subpixel registration based on intensity. Image Processing, IEEE Transactions on, 7, 27-41.

Trtik, P., Diaz, A., Guizar-Sicairos, M., Menzel, A. \& Bunk, O. (2013) Density mapping of hardened cement paste using ptychographic X-ray computed tomography. Cement and Concrete Composites, 36, 71-77.

Van Elburg, H. J., Kuypers, L. C., Decraemer, W. F. \& Dirckx, J. J. J. (2007) Improved correction of axial geometrical distortion in index-mismatched fluorescent confocal microscopic images using high-aperture objective lenses. Journal of Microscopy, 228, 45-54.

Wong, H. S. \& Buenfeld, N. R. (2006) Patch microstructure in cement-based materials: Fact or artefact? Cement and Concrete Research, 36, 990-997.

Zuiderveld, K. (1994) Contrast limited adaptive histogram equalization. In: Graphics gems IV (ed. S. H. Paul). Academic Press Professional, Inc., San Diego, United States. 